\title{
A multiphase model for chemically- and mechanically- induced cell differentiation in a hollow fibre membrane bioreactor: minimising growth factor consumption
}

\author{
Natalie C. Pearson • James M. Oliver • Rebecca J. Shipley • Sarah L. Waters
}

\begin{abstract}
We present a simplified two-dimensional mode of fluid flow, solute transport and cell distribution in a hollow fibre membrane bioreactor. We consider two cell populations, one undifferentiated and one differentiated, with differentiation stimulated by either growth factor alone, or by both growth factor and fluid shear stress. Two experimental configurations are considered, a 3-layer model in which the cells are seeded in a scaffold throughout the extracapillary space (ECS), and a 4-layer model in which the cell-scaffold construct occupies a layer surrounding the outside of the hollow fibre, only partially filling the ECS. Above this is a region of free-flowing fluid, referred to as the upper fluid layer. Following previous models by the authors (Pearson et al. 2013, 2014), we employ porous mixture theory to model the dynamics of, and interactions between, the cells, scaffold, and fluid in the cell-scaffold construct. We use this model to determine operating conditions (experiment end time, growth factor inlet concentration and inlet fluid fluxes) which result in a required percentage of differentiated cells, as well as maximising the differentiated cell yield and minimising the consumption of expensive growth factor.
\end{abstract}

Keywords tissue engineering - multiphase flow $\cdot$ cell differentiation - asymptotic reduction - fluid shear · chemical stimulation

N. C. Pearson · J. M. Oliver · S. L. Waters

OCIAM, Mathematical Institute, University of Oxford, Andrew Wiles Building, Radcliffe Observatory Quarter, Woodstock Road, Oxford, OX2 6GG, UK.

E-mail: waters@maths.ox.ac.uk

R. J. Shipley

Biomechanical Engineering Group, Department of Mechanical Engineering, University College London, Torrington Place, London WC1E 7JE, UK

\section{Introduction}

Hollow fibre membrane bioreactors (HFMBs) have great potential for use in tissue engineering, as the surface area available for seeding cells is large compared to the bioreactor volume, providing efficient mass transfer conditions (Gramer and Poeschl 2000). In addition, they have been shown to be capable of growing cells at high densities, even comparable to those of tissue in vivo (Knazek et al. 1972, 1974; Tharakan and Chau 1986). A typical setup consists of a cylindrical, glass module with a port at each end of the extracapillary space (ECS) and a porous hollow fibre inserted through the centre (see Figure 1). A number of different cell seeding and flow regimes can be employed; here, we consider the setup in which the cells are seeded in a scaffold in the ECS between the hollow fibre and the outer glass wall. This cell-scaffold construct can occupy either the entire ECS, or a layer surrounding the hollow fibre. Culture medium is pumped in via the lumen inlet and (if open) the upstream ECS port. The pressure is set at the downstream lumen end via a clamp, and the downstream ECS port is left open to the atmosphere. Depending on the flow and pressure conditions, the fluid may pass through the hollow fibre walls, and then flow out of either the lumen outlet or downstream ECS port.

Much experimental work has focussed on developing HFMBs for tissue engineering purposes, for examples, see Knazek et al. (1972); Planchamp et al. (2003); Tharakan and Chau (1986); Ye et al. (2007). However, many open research questions remain, particularly regarding how to determine optimal operating conditions (e.g. bioreactor geometries, flow rates, and supplied solute concentrations) to ensure the successful growth of a specific tissue type. 


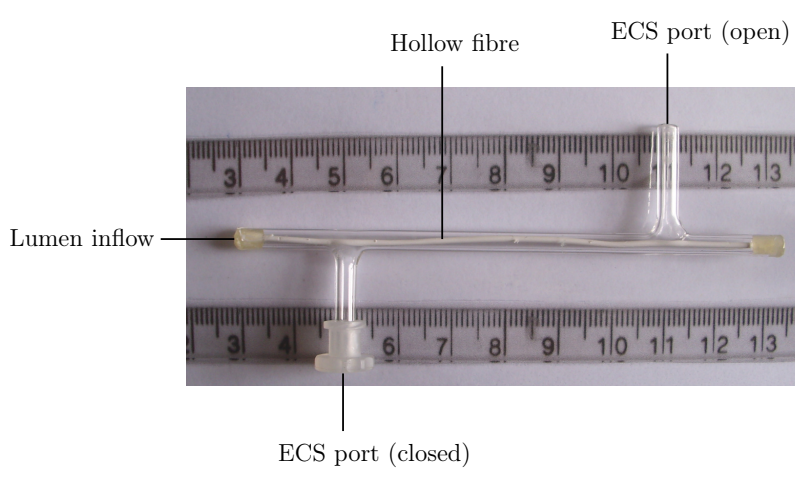

Fig. 1 Photograph of a single HFMB module (ruler scale in $\mathrm{cm}$ ) showing the ECS ports (one of which is closed off here) and the hollow fibre which runs through the centre of the module. The cell-scaffold construct can fill the entire ECS, or be in a layer surrounding the hollow fibre

Mathematical modelling of these, and other, bioreactor systems can be of great benefit for several reasons. It is reproducible and efficient, allowing the large numbers of parameters to be investigated relatively quickly and cheaply. Moreover, it can give insight into the combined effects of the physical and chemical processes involved in a particular set-up, or even focus on one process in isolation - this is hard to achieve in an experimental set-up. Existing models of HFMBs which demonstrate the power of theoretical studies include those by Shipley and co-workers: Shipley et al. (2011, 2010); Shipley and Waters (2012). Shipley et al. (2010) derived a model for the retentate and permeate flow rates in a HFMB using lubrication theory, by exploiting the small aspect ratio of the bioreactor. Theoretical values for the membrane permeability were determined and validated against experimental data. This led to the identification of equations which could be used by a tissue engineer to determine bioreactor operating conditions (e.g. flow rates, pressures, and bioreactor geometries) correlated to a specific cell-culture environment. A HFMB was also modelled by Shipley and Waters (2012), where the ECS was assumed to be seeded with cells, and fluid flow through both the porous membrane and cell-packed ECS was modelled using Darcy's law. Concentrations of both oxygen and lactate were tracked, with, respectively, a constant rate of uptake and production by the cells. The ECS ports were represented by the ECS boundary, which was treated as a single distributed port. Two experimental configurations were considered, with this 'port' either open or closed, and operating conditions were found in each case to ensure required bounds on the solute concentrations. Finally, the problem of oxygen delivery was addressed by Shipley et al. (2011). It is established that oxygen is often the limiting nutrient in tissue growth in vitro (Martin et al. 2004; Martin and Vermette 2005). By developing operating equations that linked flow rates and bioreactor geometry to critical oxygen levels in HFMBs for a variety of cell types, the authors provided tissue engineers with a way of optimizing bioreactor design to ensure sufficient oxygen delivery to cells throughout the bioreactor. Other examples of mathematical models of HFMBs include Abdullah and Das (2007); MohebbiKalhori et al. (2012) and Ye et al. (2006); for a more thorough review of the relevant literature we refer the reader to Pearson et al. (2013).

Previous papers by the authors (Pearson et al. 2013, 2014) have focussed on the yield and spatial distribution of a population of a single cell type in a HFMB, and how these measures are affected by various flow regimes and solute concentrations. This is relevant to, for instance, cell expansion experiments in which only one cell type is present. However, differentiation of one cell type into another is also required in tissue engineering setups (for example, differentiation of bone marrow mesenchymal stem cells into neuronal cells (Prabhakaran et al. 2009) and chondrocytes (Li et al. 2005)). Here we extend our previous multiphase models to include two cell populations, one undifferentiated and one differentiated. We also include the concentration of a single solute, which is taken to be a growth factor. This allows us to model cell differentiation induced both chemically (via a growth factor) and mechanically (via fluid shear stress), in the 3- and 4-layer HFMB setups of Pearson et al. (2013) and Pearson et al. (2014), respectively. We note that differentiation of certain cell types has been found to be stimulated by fluid shear stress alone, without the need for growth factors (for instance, osteoblasts (Kapur et al. 2003; Arnsdorf et al. 2009) and endothelial cells (Yamamoto et al. 2005; Obi et al. 2009)). However, as we have chosen to make growth factor consumption the focus of this paper we do not consider this case here.

Experimentally, minimising the necessary quantity of growth factor is of particular importance due to the high cost of these proteins, for example, just $100 \mu \mathrm{g}$ of bone morphogenetic protein 2 human growth factor (BMP-2) currently costs around $£ 1562^{1}$. Mathematical modelling could therefore be of use, for instance, in determining the minimum growth factor concentration needed to obtain a particular percentage/yield of differentiated cells after a certain time, or optimum operating conditions which ensure that a given percentage/yield of differentiated cells can be obtained given 
a fixed amount of available growth factor. In addition, it could be used to investigate the sensitivity of growth factor consumption to the various underlying mechanical and chemical stimuli. By varying the inlet fluid fluxes and solute concentrations, we investigate their effect on the yield of differentiated cells, and the percentage of cells which are of the differentiated phenotype. This allows us to seek operating conditions which result in a high yield and percentage of differentiated cells, but also do not consume too much expensive growth factor. We show the potential of our model, once validated, to determine the experiment end time, inlet concentration and fluid fluxes which are 'optimal' given the relative importance of cell percentage, cell yield, and growth factor consumption. To the best of our knowledge, this is the first time that cell differentiation has been modelled in a multiphase setup.

\subsection{Paper outline}

We begin by describing the model setup in $\S 2$, presenting the governing equations and boundary conditions in a simplified modelling domain. We discuss relevant dimensional and dimensionless parameter values in $\S 3.1$ and non-dimensionalise the governing equations and boundary conditions in $\S 3.2$, before considering a reduction of the 4-layer model in $\S 3.3$ and $\S 3.4$ that is appropriate when the aspect ratio of the modelling domain is small. In $\S 3.5$ we present the corresponding reduced 3layer model. Numerical results for both the 3-layer and 4 -layer models are presented in $\S 4$. In $\S 4.2$ we investigate the case where cell differentiation is dependent on growth factor concentration alone, whilst in $\S 4.3$ we consider differentiation dependent on both growth factor concentration and fluid shear stress. These dependences are incorporated via the constitutive laws for the cell mass transfer functions. Finally, we discuss our findings and conclude in $\S 5$.

\section{Model description}

As in Pearson et al. $(2013,2014)$ we model a HFMB in two-dimensional, Cartesian coordinates $(x, y)$, motivated by taking a cross section of the single bioreactor module pictured in Figure 1. In the 3-layer model, we consider the flow regime where the upstream ECS ports are closed (as depicted in Figure 1) and fluid is pumped into the lumen inlet only. In the 4-layer model, we consider the regime in which fluid is pumped in through both the lumen inlet and open upstream ECS ports. This allows us to explore the influence of the external flow in the upper fluid layer on cell differentiation. We model the dynamics in the central region of the bioreactor only, excluding the ECS ports, which allows us to make analytical progress whilst still capturing key features of the system. The effect of these excluded regions will be captured via up- and downstream boundary conditions on our reduced domain and are investigated in more detail in Pearson et al. (2014a). We also exploit the symmetry of this region and model the dynamics in the upper half of the domain only. The simplified domains for both the 3- and 4-layer models are depicted in Figure 2. As in our previous models we work in terms of reduced pressures as the effects of gravity cannot be neglected at the low flow rates we consider. We note that $H$ always denotes the height of the bioreactor, and so is given by $H=h_{1}+h_{2}+h_{3}$ in the 3-layer case, and by $H=h_{1}+h_{2}+h_{3}+h_{4}$ in the 4-layer setup.

(a)

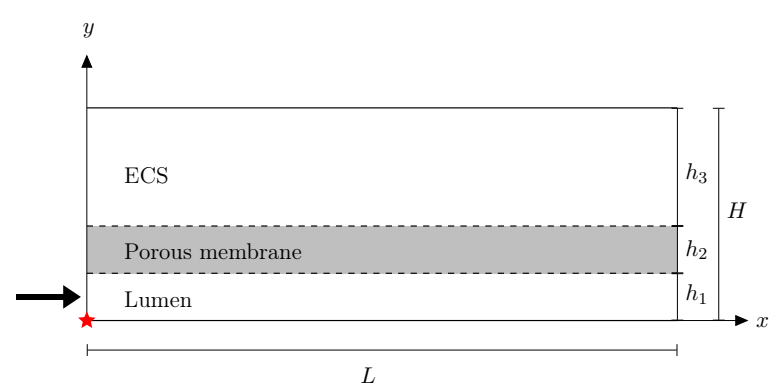

(b)

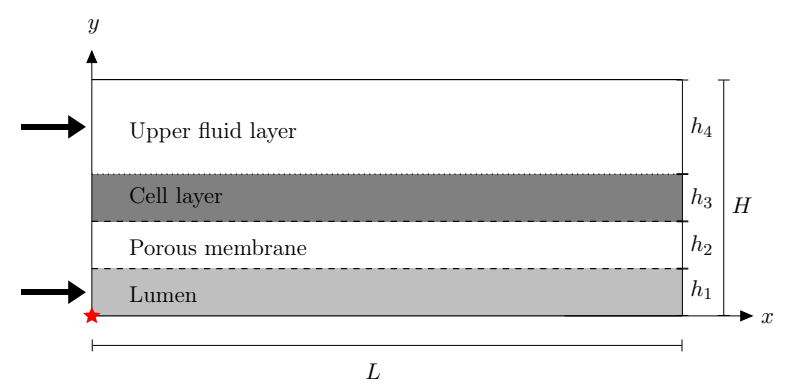

Fig. 2 Simplified modelling domains for (a) the 3-layer model, and (b) the 4-layer model, with the $x$-axis running along the lumen centreline (not to scale). In each diagram the red star denotes the origin $(x, y)=(0,0)$, and the solid black arrows denote the direction of the imposed fluid flux into the lumen and (in (b)) upper fluid layer 
Table 1 Definitions of key variables and subscripts

\begin{tabular}{ll}
\hline Subscript & Corresponding layer or phase \\
\hline $\mathrm{l}$ & lumen \\
$\mathrm{m}$ & porous membrane \\
$\mathrm{w}$ & water phase (in the ECS ${ }^{\mathrm{a}}$ or upper fluid layer $\left.{ }^{\mathrm{b}}\right)$ \\
$\mathrm{s}$ & scaffold \\
$\mathrm{u}$ & undifferentiated cell phase \\
$\mathrm{d}$ & differentiated cell phase \\
$\mathrm{f}$ & upper fluid layer ${ }^{\mathrm{b}}$ \\
\hline Variable & Definition \\
\hline $\mathbf{u}_{i}=\left(u_{i}, v_{i}\right)(i=1, \mathrm{~m}, \mathrm{w}, \mathrm{f})$ & water velocity in layer $i$ \\
$\mathbf{u}_{i}=\left(u_{i}, v_{i}\right)(i=\mathrm{u}, \mathrm{d})$ & velocity of cell phase $i$ \\
$p_{i}(i=1, \mathrm{~m}, \mathrm{w}, \mathrm{f})$ & reduced water pressure in layer $i$ \\
$p_{i}(i=\mathrm{u}, \mathrm{d})$ & reduced pressure of cell phase $i$ \\
$c_{i}(i=1, \mathrm{~m}, \mathrm{w}, \mathrm{f})$ & concentration of growth factor per unit volume of water in layer $i$ \\
$\theta_{i}(i=\mathrm{w}, \mathrm{u}, \mathrm{d}, \mathrm{s})$ & volume fraction of phase $i$ \\
\hline${ }^{a}$ 3-layer model & \\
$\mathrm{b}_{4}$-layer model &
\end{tabular}

\subsection{Governing equations}

In the lumen and upper fluid layer, we neglect inertia and the fluid flow is governed by the Stokes equations for an incompressible fluid, whilst in the porous membrane we model the flow using Darcy's law for flow in porous media. Mass transport is described by an advection-diffusion-reaction equation for the growth factor concentration. These equations are identical to those of our previous models, and hence we refer the reader to Pearson et al. (2013) and Pearson et al. (2014) for a detailed description of the dimensional systems for the 3- and 4-layer setups, respectively. We introduce the corresponding dimensionless equations following the non-dimensionalisation in $\S 3.2$. Definitions of the key variables in these layers, and in the multiphase layer, can be found in Table 1 .

In the remaining multiphase layer (the ECS for the 3 -layer setup or the cell layer for the 4-layer setup), we consider the simplest mechanical model possible to gain insight into effects caused by the differentiation stimuli. As mentioned above, we employ porous flow mixture theory to model the dynamics of, and interactions between, the four phases in the model: the water, scaffold, and undifferentiated and differentiated cell populations. The resulting equations are based on conservation of mass and momentum for each phase on the macroscale. In the cell phases, we assume that the intraphase pressure is equal to that of the water plus an extra pres- sure due to cell-cell interactions. As in Pearson et al. (2013, 2014), we assume for both cell populations that the drag between the water and cells is much smaller than that between the cells and scaffold, or water and scaffold, and that the cells cannot leave the scaffold and so remain in the ECS (3-layer model) or cell layer (4-layer model). We additionally assume that there are no tractions or drag between the two cell phases. For simplicity and motivated by our focus on cell differentiation, we neglect the effect of chemotaxis on both cell populations. The scaffold is again assumed to be inert and rigid, with its volume fraction $\theta_{\mathrm{s}}$ constant in space and time. The appropriate conservation of mass and momentum equations are analogous to those of Pearson et al. $(2013,2014)$, with the addition of an extra cell phase, and we reiterate these below.

In the ECS (3-layer system) or the cell layer (4-layer system) the no voids condition is

$\theta_{\mathrm{u}}+\theta_{\mathrm{d}}+\theta_{\mathrm{w}}+\theta_{\mathrm{s}}=1$

where we have introduced subscript notation to denote variables belonging to different phases; see Table 1 for definitions. Conservation of mass for each of the active phases yields

$$
\begin{aligned}
\frac{\partial \theta_{i}}{\partial t}+\nabla \cdot\left(\theta_{i} \mathbf{u}_{i}\right) & =J_{i} \quad(i=\mathrm{u}, \mathrm{d}), \\
\frac{\partial \theta_{\mathrm{w}}}{\partial t}+\nabla \cdot\left(\theta_{\mathrm{w}} \mathbf{u}_{\mathrm{w}}\right) & =J_{\mathrm{w}}
\end{aligned}
$$


where $t$ denotes time, and the mass transfer functions $J_{\mathrm{u}}, J_{\mathrm{d}}$, and $J_{\mathrm{w}}$ are assumed to be functions of $\theta_{\mathrm{u}}, \theta_{\mathrm{d}}$, $\theta_{\mathrm{w}}$, the fluid shear stress (through $\partial p_{\mathrm{w}} / \partial x$ as in Pearson et al. (2014)), and the growth factor concentration $c_{\mathrm{w}}$. As before we assume conservation of mass so that $J_{\mathrm{u}}+$ $J_{\mathrm{d}}+J_{\mathrm{w}}=0$.

Conservation of momentum for each phase (neglecting inertia) takes the form

$$
\begin{aligned}
-\theta_{i} \nabla p_{\mathrm{w}} & -\nabla\left(\theta_{i} \Pi_{i}\right)+\nabla \cdot\left(\theta_{i} \boldsymbol{\tau}_{i}\right) \\
& +\psi_{\mathrm{ns}} \theta_{\mathrm{s}} \nabla \theta_{i}-\gamma_{\mathrm{ns}} \theta_{i} \theta_{\mathrm{s}} \mathbf{u}_{i}=\mathbf{0} \quad(i=\mathrm{u}, \mathrm{d}), \\
& -\theta_{\mathrm{w}} \nabla p_{\mathrm{w}}-\gamma_{\mathrm{ws}} \theta_{\mathrm{w}} \theta_{\mathrm{s}} \mathbf{u}_{\mathrm{w}}=\mathbf{0}
\end{aligned}
$$

where $\Pi_{i}(i=\mathrm{u}, \mathrm{d})$ is the extra intraphase cell pressure due to cell-cell interactions (for example, osmotic stress and surface tension in the cell membranes $), \boldsymbol{\tau}_{i}(i=\mathrm{u}, \mathrm{d})$ is the cell deviatoric stress tensor and $\gamma_{\mathrm{ws}}$ is the waterscaffold drag coefficient. We have also introduced the extra interphase pressure term $\psi_{\text {ns }}$ due to cell-scaffold tractions and the cell-scaffold drag coefficient $\gamma_{\mathrm{ns}}$, both of which are assumed to be the same for each cell population (although it is straightforward to modify the model to account for differences in these parameters between the cell populations). We have assumed that the two cell populations have the same effective viscosity $\mu_{\mathrm{n}}$. We note that this assumption could easily be relaxed, and would involve carrying around an extra parameter representing the ratio of the two effective viscosities in the dimensionless system. A more detailed discussion of the momentum equations in (2.4) can be found in Pearson et al. (2013).

We must further specify constitutive forms for $\psi_{\mathrm{ns}}$ and $\Pi_{i}(i=\mathrm{u}, \mathrm{d})$. The extra interphase pressure $\psi_{\mathrm{ns}}$ is assumed to be a negative constant representing the cells' affinity for the scaffold, so that

$\psi_{\mathrm{ns}}=-\eta$

The extra intraphase pressure term $\Pi_{i}(i=\mathrm{u}, \mathrm{d})$ for each cell population is assumed to take the form (see Pearson et al. (2013); O'Dea et al. (2010))

$$
\Pi_{i}=\theta_{i}\left(-\nu+\frac{\delta_{\mathrm{a}} \theta_{i}}{1-\theta_{\mathrm{s}}-\theta_{i}-\theta_{j}}\right) \quad(i, j=\mathrm{u}, \mathrm{d}, i \neq j),
$$

where $\nu$ and $\delta_{\mathrm{a}}(i=\mathrm{u}, \mathrm{d})$ are constants and we have again assumed that they are the same for each cell population.

Finally, we model mass transport in the multiphase layer with an advection-reaction-diffusion equation,

$$
\frac{\partial}{\partial t}\left(\theta_{\mathrm{w}} c_{\mathrm{w}}\right)+\nabla \cdot\left(\theta_{\mathrm{w}} c_{\mathrm{w}} \mathbf{u}_{\mathrm{w}}\right)=D \nabla \cdot\left(\theta_{\mathrm{w}} \nabla c_{\mathrm{w}}\right)+\mathcal{R}
$$

where the reaction term $\mathcal{R}$ depends on any of $\theta_{\mathrm{u}}, \theta_{\mathrm{d}}$, and $c_{\mathrm{w}}$ and captures uptake of solute by the cells, and $D$ is the diffusion coefficient of the growth factor in water (assumed constant and to take the same value in each layer). We note here that growth factors are proteins, which are typically much larger than nutrient molecules such as oxygen. Hence it could be argued that a Fickian diffusion term is unsuitable, and an approach taking into account multi-component effects (e.g. interactions between solute molecules) would be more appropriate. Abdullah and Das include these effects via MaxwellStefan equations in their model of nutrient transport in a HFMB (Abdullah and Das 2007), and find that they can be of significance in reducing mass transfer in certain experimental configurations: sufficiently high inlet concentrations, solute molecular sizes, and ECS and fibre wall thicknesses. However, inclusion of such interactions necessitates a much more complicated modelling framework, involving additional parameter values which are currently unknown or difficult to interpret physically. We therefore neglect these effects in our first cell differentiation model and assume a Fickian diffusion term for the growth factor concentration, an approach also taken in, for example, Brouwers et al. (2006); Byrne et al. (2006); Plank et al. (2004).

It remains to prescribe constitutive forms for $J_{\mathrm{u}}$, $J_{\mathrm{d}}$, and $\mathcal{R}$; to keep our analysis general we delay their specification until results are obtained in $\S 4$.

\subsection{Boundary conditions}

We first discuss the boundary conditions applied to the fluid and solute throughout the modelling domain. On the lumen centreline we impose symmetry and no flux of fluid or of solute. On the lumen/membrane interface, we impose no slip and continuity of fluid flux, of fluid normal stress, of concentration and of solute flux. The appropriate boundary conditions on the membrane/ECS (3-layer model) or membrane/cell layer (4layer model) interface are continuity of fluid flux, of fluid normal stress, of concentration and of solute flux. On the bioreactor boundary we impose no flux of fluid or of solute. We again introduce all the above conditions in their equation form in $\S 3.3$ after we have nondimensionalised, along with the up- and downstream conditions at the inlets and outlets.

We now present the boundary conditions applied to the cell phases, which are analogous to the corresponding single-cell-population models in Pearson et al. (2013) and Pearson et al. (2014). On the membrane/ECS (3-layer model) or membrane/cell layer (4-layer model) 
interface we impose no flux and no slip of cells,

$\mathbf{u}_{i}=\mathbf{0} \quad(i=\mathrm{u}, \mathrm{d}) \quad$ on $\quad y=h_{1}+h_{2}$.

In addition, on the bioreactor top (3-layer model) we also have no flux and no slip of cells,

$\mathbf{u}_{i}=\mathbf{0} \quad(i=\mathrm{u}, \mathrm{d}) \quad$ on $\quad y=H$,

and on the cell layer/upper fluid layer interface (4-layer model) we impose no flux and no shear stress on the cell phase,

$$
\begin{aligned}
v_{i}=0, \quad \mathbf{n}_{\mathrm{e}} \cdot \boldsymbol{\sigma}_{i} \cdot \mathbf{t}_{\mathrm{e}} & =0 \quad(i=\mathrm{u}, \mathrm{d}) \\
\text { on } \quad y & =H-h_{4},
\end{aligned}
$$

where $\mathbf{n}_{\mathrm{e}}$ and $\mathbf{t}_{\mathrm{e}}$ are the unit normal (positive $y$-direction) and unit tangent to the cell layer/upper fluid layer interface, respectively.

We also impose no flux of both cell populations out of the multiphase region at $x=0, L$, and introduce this along with the other up- and downstream conditions as required in $\S 3.3$.

\section{Model reduction}

We now discuss dimensional and dimensionless parameter values before non-dimensionalising and reducing the 4-layer model to a coupled system of three PDEs. We then summarise the corresponding system of four coupled PDEs for the 3-layer model.

\subsection{Parameter values}

We take the majority of the dimensional parameter values to be the same as in our previous multiphase models in Pearson et al. (2013, 2014), and only discuss in detail below those parameters referring to the solute and cell populations which are different to previous work. Dimensional parameter values for which data are available are given in Table 2 and all dimensionless parameters are given and defined in Table 3. The key difference between this model and those in Pearson et al. $(2013,2014)$ is that the solute of interest here is a growth factor. These molecules are much larger than oxygen, and we therefore take a representative value for $D$ of $2 \times 10^{-10} \mathrm{~m}^{2} \mathrm{~s}^{-1}$, based on an estimate for the diffusion coefficient for vascular endothelial growth factor in aqueous solution (Mac Gabhann et al. 2005). This results in a reduced Péclet number of 0.0102 , so that $\varepsilon^{2} \ll \varepsilon^{2} \mathrm{Pe} \ll 1$, where $\varepsilon=h_{1} / L$ is the lumen aspect ratio, and radial diffusion dominates advection throughout. Hence we are in a different distinguished limit to Pearson et al. (2013, 2014) when considering the system of equations governing the solute concentration. The typical concentration used to set the scaling $C^{*}$ is larger for growth factors than for oxygen; we choose $C^{*}=7.69 \mathrm{~mol} \mathrm{~m}^{-3}$, a typical inlet concentration of BMP-2, following discussions with our experimental collaborators $^{2,3}$. In $\S 4$ we investigate the effect of varying the inlet growth factor concentration on the yield of the cell populations by considering a range of values of $c_{\text {in }}$. We choose the reaction scale $R^{*}=C^{*} D /\left(\varepsilon L^{2}\right)$ so that solute uptake appears in our leading-order model (see $\S 3.3)$.

The dimensionless values of the constants appearing in the drag and pressure terms for the cell populations, $\hat{\nu}, \hat{\delta}_{\mathrm{a}}$ and $\hat{\eta}$ are set to be the same as the single-cellpopulation models in Pearson et al. (2013) and Pearson et al. (2014), as is the dimensionless cell-scaffold drag parameter $\zeta_{\text {ns. }}$ In the 4-layer model, we take the dimensionless cell layer height $\hat{h}_{3}$ (and therefore also $\hat{h}_{4}$ ) to be of $\mathrm{O}(1)$.

When obtaining the numerical results in $\S 4$, we fix a maximum end time $T$ and look at the percentage and yield of cells which are of the differentiated type within this time range. A typical length of the 'differentiation phase' of an experiment is $2-4$ weeks $^{2}$, and so we set $T$ to be the upper value of 4 weeks, corresponding to a dimensionless end time of 0.2477 (given the timescale $L / U^{*}$ introduced in $\S 3.2$ ).

In the case studies that follow in $\S 4$, we also need to specify parameters within the constitutive forms for $J_{\mathrm{u}}, J_{\mathrm{d}}$, and $\mathcal{R}$. The cell death rate $\Gamma_{\mathrm{wn}}$ (assumed for simplicity to be the same for both populations) is chosen assuming that cells live, on average, for 28 days $^{2}$, corresponding to a dimensionless value of 4.04. The cell proliferation rate, $\Gamma_{\mathrm{nw}}$, is also assumed to be the same for both populations and is taken to have a lower dimensional value than in our previous models (see below for the exact choice of value), as would be expected in the differentiation phase of an experiment ${ }^{2}$.

The remaining parameters are the cell differentiation rate $\Gamma_{\mathrm{ud}}$, the solute uptake rate $\Gamma_{\mathrm{R} 1}$, and the Michaelis-Menten uptake constant $K$. Given that experimental data for these parameters are not available, their dimensionless equivalents (which are defined in Table 3) are chosen to be of $\mathrm{O}(1)$ for illustrative purposes, so that we retain as many effects as possible in our leading-order model. In addition, values are assigned according to two experimental observations ${ }^{2}$ : (1) the cell differentiation rate is much faster than the cell proliferation rate (in the differentiation phase of experiments which we are modelling), and (2) at the end of an experiment almost all $(98-99 \%)$ cells are of the differentiated type. We use the second observation to determine candidate values for $\Gamma_{\mathrm{ud}}, \Gamma_{\mathrm{R} 1}, \Gamma_{\mathrm{nw}}$, and $K$, 
Table 2 Dimensional parameters and definitions

\begin{tabular}{lll}
\hline Parameter & Dimensional value and units & Definition \\
\hline$h_{1}$ & $200 \mu \mathrm{m}^{\mathrm{a}}$ & lumen height \\
$h_{2}$ & $200 \mu \mathrm{m}^{\mathrm{a}}$ & porous membrane height \\
$h_{3}$ & $600 \mu \mathrm{m}^{\mathrm{a}}$ & ECS height \\
$h_{3}+h_{4}$ & $600 \mu \mathrm{m}^{\mathrm{a}}$ & ECS height \\
$L$ & $0.1 \mathrm{~m}^{\mathrm{a}}$ & length of modelling domain \\
$\rho_{\mathrm{w}}$ & $1 \mathrm{~g} \mathrm{~cm}^{-3} \mathrm{a}$ & water density \\
$\mu_{\mathrm{w}}$ & $10^{-3} \mathrm{~Pa} \mathrm{~s}^{\mathrm{a}}$ & water viscosity \\
$k_{\mathrm{m}}$ & $6.73 \times 10^{-16} \mathrm{~m}^{2 \mathrm{e}}$ & porous membrane permeability \\
$p_{\mathrm{atm}}$ & $14.69 \mathrm{psia}^{\mathrm{a}}$ & atmospheric pressure \\
$Q_{\mathrm{l}, \mathrm{in}}, Q_{\mathrm{f}, \mathrm{in}}$ & $1.02 \times 10^{-11}-1.02 \times 10^{-8} \mathrm{~m}^{2} \mathrm{~s}^{-1} \mathrm{f}$ & lumen/upper fluid layer inlet flux \\
$\Gamma_{\mathrm{wn}}$ & $4.13 \times 10^{-7} \mathrm{~s}^{-1 \mathrm{f}}$ & cell death rate coefficient \\
$U^{*}$ & $1.0239 \times 10^{-8} \mathrm{~m} \mathrm{~s}^{-1 \mathrm{f}}$ & typical porous membrane velocity \\
$D$ & $2 \times 10^{-10} \mathrm{~m}^{2} \mathrm{~s}^{-1 \mathrm{~b}}$ & growth factor diffusion coefficient in water \\
$C^{*}$ & $7.69 \mathrm{~mol} \mathrm{~m}^{-3} \mathrm{f}$ & typical growth factor concentration \\
$T$ & $2.42 \times 10^{6} \mathrm{~s}^{\mathrm{f}}$ & maximum experiment end time \\
\hline
\end{tabular}

a Values taken from Shipley et al. (2010)

b Value taken from Mac Gabhann et al. (2005)

c 3-layer model

d 4-layer model

e Value obtained experimentally

$\mathrm{f}$ Values based on estimations by our experimental collaborators

we first set the other parameters whose values can vary to the following fixed values: $Q_{1, \text { in }}=1, Q_{\mathrm{f} \text {,in }}=0.1$, $c_{\mathrm{in}}=1$. The dimensionless values for $\Gamma_{\mathrm{nw}}, \Gamma_{\mathrm{ud}}, \Gamma_{\mathrm{R} 1}$, and $K$ given in Table 3 are then chosen so that (to two significant figures) $99 \%$ of cells were of the differentiated type at the maximum end time $T$ when solving the case study from $\S 4.2$ (3- and 4-layer models).

\subsection{Non-dimensionalisation}

With the exception of the reaction scale $R^{*}$ (as discussed in §3.1), we non-dimensionalise with the same scalings as in our previous models, assuming that the two cell populations have the same velocity scale:

$$
\begin{gathered}
x=L \hat{x}, \quad y=\varepsilon L \hat{y}, \quad t=\frac{L}{U^{*}} \hat{t}, \\
h_{i}=\varepsilon L \hat{h}_{i} \quad(i=2,3,4), \quad H=\varepsilon L \hat{H}, \\
u_{i}=\frac{\mu_{\mathrm{n}}}{\mu_{\mathrm{w}}} U^{*} \hat{u}_{i}, \quad v_{i}=\frac{\varepsilon \mu_{\mathrm{n}}}{\mu_{\mathrm{w}}} U^{*} \hat{v}_{i} \quad(i=1, \mathrm{f}), \\
u_{i}=U^{*} \hat{u}_{i}, \quad v_{i}=\varepsilon U^{*} \hat{v}_{i} \quad(i=\mathrm{m}, \mathrm{w}, \mathrm{u}, \mathrm{d}), \\
p_{i}=p_{\mathrm{atm}}+\frac{\mu_{\mathrm{n}} U^{*}}{\varepsilon^{2} L} \hat{p}_{i} \quad(i=1, \mathrm{~m}, \mathrm{w}, \mathrm{f}), \\
\Pi_{i}=\frac{\mu_{\mathrm{n}} U^{*}}{\varepsilon^{2} L} \hat{\Pi}_{i} \quad(i=\mathrm{u}, \mathrm{d}), \quad \psi_{\mathrm{ns}}=\frac{\mu_{\mathrm{n}} U^{*}}{\varepsilon^{2} L} \hat{\psi}_{\mathrm{ns}}, \\
\mathcal{R}=\frac{D C^{*}}{\varepsilon L^{2}} \hat{\mathcal{R}}, \quad C_{i}=\frac{U^{*}}{L} \hat{J}_{i} \quad(i=\mathrm{u}, \mathrm{d}) .
\end{gathered}
$$

We substitute these scalings into just the multiphase equations for now; the corresponding dimensionless equations in the lumen, porous membrane and (for the 4layer model) the upper fluid layer can be found in Appendix A. Dropping hats on dimensionless variables, this yields for the cell phases

$$
\frac{\partial \theta_{i}}{\partial t}+\frac{\partial}{\partial x}\left(\theta_{i} u_{i}\right)+\frac{\partial}{\partial y}\left(\theta_{i} v_{i}\right)=J_{i} \quad(i=\mathrm{u}, \mathrm{d})
$$


Table 3 Dimensionless parameters, along with their definitions and any bounds imposed either physically or by the asymptotic analysis

\begin{tabular}{|c|c|c|c|}
\hline Parameter & Definition & Value & Restriction \\
\hline$\varepsilon$ & $h_{1} / L$ & $2 \times 10^{-3}$ & $0<\varepsilon \ll 1$ \\
\hline$\hat{h}_{2}$ & $h_{2} /(\varepsilon L)$ & 1 & $\hat{h}_{2}>0$ \\
\hline$\hat{h}_{3}$ & $h_{3} /(\varepsilon L)$ & $3^{\mathrm{a}}$ & $\hat{h}_{3}>0$ \\
\hline$\hat{h}_{3}+\hat{h}_{4}$ & $\left(h_{3}+h_{4}\right) /(\varepsilon L)$ & $3^{\mathrm{b}}$ & $\hat{h}_{3}, \hat{h}_{4}>0$ \\
\hline$\theta_{\mathrm{s}}$ & Scaffold volume fraction & $0.4^{\mathrm{c}}$ & $0<\theta_{\mathrm{s}}<1$ \\
\hline$\lambda$ & $\mu_{\mathrm{w}} /\left(\varepsilon \mu_{\mathrm{n}}\right)$ & 1 & $\varepsilon \ll \lambda \ll 1 / \varepsilon$ \\
\hline$\varepsilon^{2} \mathrm{Pe}$ & $\varepsilon L U^{*} /(\lambda D)$ & 0.0102 & $\varepsilon^{2} \ll \varepsilon^{2} \mathrm{Pe} \ll 1$ \\
\hline$\varepsilon^{2} \operatorname{Re}$ & $\varepsilon \rho_{\mathrm{w}} L U^{*} /\left(\lambda \mu_{\mathrm{w}}\right)$ & $2.05 \times 10^{-6}$ & $\varepsilon^{2} \operatorname{Re} \ll 1$ \\
\hline$\phi_{\mathrm{m}}$ & Porous membrane porosity & $0.77^{\mathrm{d}}$ & $0<\phi_{\mathrm{m}}<1$ \\
\hline$\kappa_{\mathrm{m}}$ & $k_{\mathrm{m}} /\left(\lambda \varepsilon^{5} L^{2}\right)$ & 2.1 & $\varepsilon \ll \kappa_{\mathrm{m}} \ll 1 / \varepsilon$ \\
\hline$\hat{Q}_{i, \text { in }}(i=1, \mathrm{f})$ & $\lambda Q_{i, \text { in }} /\left(L U^{*}\right)$ & $0.01-10$ & $\varepsilon \ll \hat{Q}_{i, \text { in }} \ll 1 / \varepsilon$ \\
\hline$\hat{\nu}$ & $\lambda \varepsilon^{3} L \nu /\left(\mu_{w} U^{*}\right)$ & $0.3^{\mathrm{e}}$ & $\varepsilon \ll \hat{\nu} \ll 1 / \varepsilon$ \\
\hline$\hat{\delta}_{\mathrm{a}}$ & $\lambda \varepsilon^{3} L \delta_{\mathrm{a}} /\left(\mu_{w} U^{*}\right)$ & $0.1^{\mathrm{e}}$ & $\varepsilon \ll \hat{\delta}_{\mathrm{a}} \ll 1 / \varepsilon$ \\
\hline$\hat{\eta}$ & $\lambda \varepsilon^{3} L \eta /\left(\mu_{w} U^{*}\right)$ & $0.3^{\mathrm{e}}$ & $\varepsilon \ll \hat{\eta} \ll 1 / \varepsilon$ \\
\hline$\zeta_{\mathrm{ns}}$ & $\gamma_{\mathrm{ns}} L^{2} \lambda \varepsilon^{3} / \mu_{\mathrm{w}}$ & 1 & $\varepsilon \ll \zeta_{\mathrm{ns}} \ll 1 / \varepsilon$ \\
\hline$\zeta_{\mathrm{ws}}$ & $\gamma_{\mathrm{ws}} L^{2} \lambda \varepsilon^{3} / \mu_{\mathrm{w}}$ & 0.1 & $\varepsilon \ll \zeta_{\mathrm{ws}} \ll 1 / \varepsilon$ \\
\hline$\hat{\Gamma}_{\mathrm{nw}}$ & $L \Gamma_{\mathrm{nw}} / U^{*}$ & 6 & $\varepsilon \ll \hat{\Gamma}_{\mathrm{nw}} \ll 1 / \varepsilon$ \\
\hline$\hat{\Gamma}_{\text {wn }}$ & $L \Gamma_{\mathrm{wn}} / U^{*}$ & 4.04 & $\varepsilon \ll \hat{\Gamma}_{\text {wn }} \ll 1 / \varepsilon$ \\
\hline$\hat{\Gamma}_{\mathrm{ud}}$ & $L \Gamma_{\mathrm{ud}} / U^{*}$ & 70 & $\varepsilon \ll \hat{\Gamma}_{\mathrm{nw}} \ll 1 / \varepsilon$ \\
\hline$\hat{\Gamma}_{\mathrm{R} 1}$ & $\varepsilon L^{2} \Gamma_{\mathrm{R} 1} / D$ & 1 & $\varepsilon \ll \hat{\Gamma}_{\mathrm{R} 1} \ll 1 / \varepsilon$ \\
\hline$\hat{K}$ & $K / C^{*}$ & 0.4 & $\varepsilon \ll \hat{K} \ll 1 / \varepsilon$ \\
\hline$\hat{c}_{\text {in }}$ & $c_{\mathrm{in}} / C^{*}$ & $0.1-1$ & $\varepsilon \ll \hat{c}_{\text {in }} \ll 1 / \varepsilon$ \\
\hline$\hat{P}_{\text {dwn }}$ & $\varepsilon^{2} L\left(P_{\mathrm{dwn}}-p_{\mathrm{atm}}\right) /\left(\mu_{\mathrm{n}} U^{*}\right)$ & 2.5 & $\varepsilon \ll \hat{P}_{\mathrm{d}} \ll 1 / \varepsilon$ \\
\hline$\hat{T}$ & $U^{*} T / L$ & 0.2477 & - \\
\hline \multicolumn{4}{|l|}{ a 3-layer model. } \\
\hline \multicolumn{4}{|l|}{ b 4-layer model. } \\
\hline \multicolumn{4}{|c|}{${ }^{\mathrm{c}}$ Value taken from Lemon et al. (2006) } \\
\hline \multicolumn{4}{|c|}{${ }^{\mathrm{d}}$ Value taken from Meneghello et al. (2009) } \\
\hline e Values taken $\mathrm{fr}$ & n O'Dea et al. (2010) & & \\
\hline
\end{tabular}

and

$$
\begin{gathered}
-\theta_{i} \frac{\partial p_{\mathrm{w}}}{\partial x}-\frac{\partial}{\partial x}\left(\theta_{i} \Pi_{i}\right)+\frac{2 \varepsilon^{2}}{3} \frac{\partial}{\partial x}\left[\theta_{i}\left(2 \frac{\partial u_{i}}{\partial x}-\frac{\partial v_{i}}{\partial y}\right)\right] \\
+\frac{\partial}{\partial y}\left[\theta_{i}\left(\frac{\partial u_{i}}{\partial y}+\varepsilon^{2} \frac{\partial v_{i}}{\partial x}\right)\right]+\psi_{\mathrm{ns}} \theta_{\mathrm{s}} \frac{\partial \theta_{i}}{\partial x} \\
-\zeta_{\mathrm{ns}} \theta_{i} \theta_{\mathrm{s}} u_{i}=0 \quad(i=\mathrm{u}, \mathrm{d}), \\
-\theta_{i} \frac{\partial p_{\mathrm{w}}}{\partial y}-\frac{\partial}{\partial y}\left(\theta_{i} \Pi_{i}\right)+\varepsilon^{2} \frac{\partial}{\partial x}\left[\theta_{i}\left(\frac{\partial u_{i}}{\partial y}+\varepsilon^{2} \frac{\partial v_{i}}{\partial x}\right)\right] \\
+\frac{2 \varepsilon^{2}}{3} \frac{\partial}{\partial y}\left[\theta_{i}\left(2 \frac{\partial v_{i}}{\partial y}-\frac{\partial u_{i}}{\partial x}\right)\right]+\psi_{\mathrm{ns}} \theta_{\mathrm{s}} \frac{\partial \theta_{i}}{\partial y} \\
-\varepsilon^{2} \zeta_{\mathrm{ns}} \theta_{i} \theta_{\mathrm{s}} v_{i}=0 \quad(i=\mathrm{u}, \mathrm{d}),
\end{gathered}
$$

and for the water phase

$$
\begin{aligned}
\frac{\partial \theta_{\mathrm{w}}}{\partial t}+\frac{\partial}{\partial x}\left(\theta_{\mathrm{w}} u_{\mathrm{w}}\right)+\frac{\partial}{\partial y}\left(\theta_{\mathrm{w}} v_{\mathrm{w}}\right) & =J_{\mathrm{w}} \\
& =-\left(J_{\mathrm{u}}+J_{\mathrm{d}}\right), \\
-\theta_{\mathrm{w}} \frac{\partial p_{\mathrm{w}}}{\partial x}-\zeta_{\mathrm{ws}} \theta_{\mathrm{w}} \theta_{\mathrm{s}} u_{\mathrm{w}} & =0 \\
-\theta_{\mathrm{w}} \frac{\partial p_{\mathrm{w}}}{\partial y}-\varepsilon^{2} \zeta_{\mathrm{ws}} \theta_{\mathrm{w}} \theta_{\mathrm{s}} v_{\mathrm{w}} & =0
\end{aligned}
$$

where $\zeta_{i \mathrm{~s}}=\gamma_{i \mathrm{~s}} L^{2} \varepsilon^{2} / \mu_{\mathrm{n}}(i=\mathrm{w}, \mathrm{n})$ is the dimensionless water-scaffold/cell-scaffold drag. The dimensionless 
mass transport equation is

$$
\begin{aligned}
\lambda \varepsilon^{3} \operatorname{Pe} & \left(\frac{\partial}{\partial t}\left(\theta_{\mathrm{w}} c_{\mathrm{w}}\right)+\nabla \cdot\left(\theta_{\mathrm{w}} c_{\mathrm{w}} \mathbf{u}_{\mathrm{w}}\right)\right) \\
= & \varepsilon^{2} \frac{\partial}{\partial x}\left(\theta_{\mathrm{w}} \frac{\partial c_{\mathrm{w}}}{\partial x}\right)+\frac{\partial}{\partial y}\left(\theta_{\mathrm{w}} \frac{\partial c_{\mathrm{w}}}{\partial y}\right)+\varepsilon \mathcal{R} .
\end{aligned}
$$

The dimensionless boundary conditions for the cell phases are

$$
\begin{aligned}
u_{i}=v_{i}=0 \quad(i=\mathrm{u}, \mathrm{d}) & \text { on } y=1+h_{2}, \\
u_{i}=v_{i}=0 \quad(i=\mathrm{u}, \mathrm{d}) \quad & \text { on } y=H, \\
v_{i}=0, \quad \frac{\partial u_{i}}{\partial y}+\varepsilon^{2} \frac{\partial v_{i}}{\partial x}=0 & \text { on } y=H-h_{4},
\end{aligned}
$$

where (3.10) applies to the 3-layer model and (3.11) to the 4-layer model.

\subsection{Derivation of the reduced 4-layer model}

The asymptotic analysis for the 4-layer model is very similar to that in Pearson et al. (2014) and results in a coupled system of three PDEs which can be solved numerically. Below we briefly present the reduced model derivation. The 3-layer model can be reduced in an analogous way, and we therefore omit the detail but summarise the resulting system of four coupled PDEs in $\S 3.5$.

We expand all dependent variables in powers of the lumen aspect ratio $\varepsilon$, setting $u_{1} \sim u_{\mathrm{l}_{0}}+\varepsilon u_{\mathrm{l}_{1}}+\varepsilon^{2} u_{\mathrm{l}_{2}}+\ldots$ and similarly for the remaining velocities $v_{\mathrm{l}}, u_{i}, v_{i}(i=$ $\mathrm{m}, \mathrm{w}, \mathrm{u}, \mathrm{d}, \mathrm{f})$, the reduced pressures $p_{i}(i=\mathrm{l}, \mathrm{m}, \mathrm{w}, \mathrm{f})$, the concentrations $c_{i}(i=\mathrm{l}, \mathrm{m}, \mathrm{w}, \mathrm{f})$, and the volume fractions $\theta_{\mathrm{u}}, \theta_{\mathrm{d}}$ and $\theta_{\mathrm{w}}$. We then collect coefficients of $\varepsilon^{0}$ in each of the equations in $\S 3.2$ and Appendix $\mathrm{A}$ to obtain the leading-order system in each layer. Beginning in the lumen $(0<y<1)$ and omitting the subscript 0 from the leading-order variables, the $\mathrm{O}(1)$ equations are

$$
\begin{gathered}
\frac{\partial u_{1}}{\partial x}+\frac{\partial v_{1}}{\partial y}=0, \quad \frac{\partial^{2} u_{1}}{\partial y^{2}}=\frac{\partial p_{1}}{\partial x} \\
\frac{\partial p_{1}}{\partial y}=0, \quad \frac{\partial^{2} c_{1}}{\partial y^{2}}=0
\end{gathered}
$$

and in the porous membrane $\left(1<y<1+h_{2}\right)$

$$
\begin{gathered}
u_{\mathrm{m}} \equiv 0, \quad v_{\mathrm{m}}=-\kappa_{\mathrm{m}} \frac{\partial p_{\mathrm{m}}}{\partial y} \\
\frac{\partial^{2} p_{\mathrm{m}}}{\partial y^{2}}=0, \quad \frac{\partial^{2} c_{\mathrm{m}}}{\partial y^{2}}=0
\end{gathered}
$$

where $\kappa_{\mathrm{m}}=k_{\mathrm{m}} /\left(\lambda \varepsilon^{5} L^{2}\right)$. In the cell layer $\left(1+h_{2}<y<\right.$ $\left.H-h_{4}\right)$ the no voids condition is

$\theta_{\mathrm{u}}+\theta_{\mathrm{d}}+\theta_{\mathrm{w}}+\theta_{\mathrm{s}}=1$

the cell phase equations are

$$
\begin{aligned}
& \frac{\partial \theta_{i}}{\partial t}+\frac{\partial}{\partial x}\left(\theta_{i} u_{i}\right)+\frac{\partial}{\partial y}\left(\theta_{i} v_{i}\right)=J_{i} \quad(i=\mathrm{u}, \mathrm{d}) \\
& -\theta_{i} \frac{\partial p_{\mathrm{w}}}{\partial x}-\frac{\partial}{\partial x}\left(\theta_{i} \Pi_{i}\right)+\psi_{\mathrm{ns}} \theta_{\mathrm{s}} \frac{\partial \theta_{i}}{\partial x} \\
& -\zeta_{\mathrm{ns}} \theta_{i} \theta_{\mathrm{s}} u_{i}+\frac{\partial}{\partial y}\left(\theta_{i} \frac{\partial u_{i}}{\partial y}\right)=0 \quad(i=\mathrm{u}, \mathrm{d}) \\
& -\theta_{i} \frac{\partial p_{\mathrm{w}}}{\partial y}-\frac{\partial}{\partial y}\left(\theta_{i} \Pi_{i}\right)+\psi_{\mathrm{ns}} \theta_{\mathrm{s}} \frac{\partial \theta_{i}}{\partial y}=0 \quad(i=\mathrm{u}, \mathrm{d})
\end{aligned}
$$

the water phase equations are

$$
\begin{aligned}
\frac{\partial \theta_{\mathrm{w}}}{\partial t}+\frac{\partial}{\partial x}\left(\theta_{\mathrm{w}} u_{\mathrm{w}}\right)+\frac{\partial}{\partial y}\left(\theta_{\mathrm{w}} v_{\mathrm{w}}\right) & =J_{\mathrm{w}} \\
& =-J_{\mathrm{u}}-J_{\mathrm{d}},
\end{aligned}
$$

$-\theta_{\mathrm{w}} \frac{\partial p_{\mathrm{w}}}{\partial x}-\zeta_{\mathrm{ws}} \theta_{\mathrm{w}} \theta_{\mathrm{s}} u_{\mathrm{w}}=0, \quad-\theta_{\mathrm{w}} \frac{\partial p_{\mathrm{w}}}{\partial y}=0$,

and the solute equation is

$$
\frac{\partial}{\partial y}\left(\theta_{\mathrm{w}} \frac{\partial c_{\mathrm{w}}}{\partial y}\right)=0
$$

Finally, the leading-order equations in the upper fluid layer $\left(H-h_{4}<y<H\right)$ are

$$
\begin{gathered}
\frac{\partial u_{\mathrm{f}}}{\partial x}+\frac{\partial v_{\mathrm{f}}}{\partial y}=0, \quad \frac{\partial^{2} u_{\mathrm{f}}}{\partial y^{2}}=\frac{\partial p_{\mathrm{f}}}{\partial x}, \\
\frac{\partial p_{\mathrm{f}}}{\partial y}=0, \quad \frac{\partial^{2} c_{\mathrm{f}}}{\partial y^{2}}=0 .
\end{gathered}
$$

The leading-order boundary conditions on the lumen centreline are

$\frac{\partial u_{1}}{\partial y}=0, \quad v_{1}=0, \quad \frac{\partial c_{1}}{\partial y}=0 \quad$ on $\quad y=0$

on the lumen/porous membrane interface

$$
\begin{gathered}
u_{\mathrm{l}}=v_{\mathrm{l}}=0, \quad p_{\mathrm{l}}=p_{\mathrm{m}}, \\
c_{\mathrm{l}}=c_{\mathrm{m}}, \quad \frac{\partial c_{\mathrm{l}}}{\partial y}=\phi_{\mathrm{m}} \frac{\partial c_{\mathrm{m}}}{\partial y} \quad \text { on } \quad y=1,
\end{gathered}
$$

on the porous membrane/cell layer interface

$$
\begin{gathered}
u_{i}=v_{i}=0 \quad(i=\mathrm{u}, \mathrm{d}) \\
v_{\mathrm{w}}=-\frac{\kappa_{\mathrm{m}} \phi_{\mathrm{m}}}{\theta_{\mathrm{w}}} \frac{\partial p_{\mathrm{m}}}{\partial y}, \quad p_{\mathrm{m}}=p_{\mathrm{w}}, \\
c_{\mathrm{m}}=c_{\mathrm{w}}, \quad \phi_{\mathrm{m}} \frac{\partial c_{\mathrm{m}}}{\partial y}=\theta_{\mathrm{w}} \frac{\partial c_{\mathrm{w}}}{\partial y} \quad \text { on } \quad y=1+h_{2},
\end{gathered}
$$


$(3.24 a-f)$

on the cell layer/upper fluid layer interface

$$
\begin{gathered}
\frac{\partial u_{i}}{\partial y}=0, \quad v_{i}=0 \quad(i=\mathrm{u}, \mathrm{d}), \\
u_{\mathrm{f}}=v_{\mathrm{f}}=0, \quad p_{\mathrm{w}}=p_{\mathrm{f}}, \\
c_{\mathrm{w}}=c_{\mathrm{f}}, \quad \theta_{\mathrm{w}} \frac{\partial c_{\mathrm{w}}}{\partial y}=\frac{\partial c_{\mathrm{f}}}{\partial y} \quad \text { on } \quad y=H-h_{4},
\end{gathered}
$$

and on the bioreactor top

$u_{\mathrm{f}}=v_{\mathrm{f}}=0, \quad \frac{\partial c_{\mathrm{f}}}{\partial y}=0 \quad$ on $\quad y=H$.

We also now introduce the up- and downstream boundary conditions on the fluid velocities and pressures in the lumen and upper fluid layer. These are identical to those in Pearson et al. (2014). We prescribe the inlet fluid fluxes and outlet pressures in both the lumen and upper fluid layer, setting

$Q_{1, \text { in }}=\int_{0}^{1} u_{\mathrm{l}} \mathrm{d} y, \quad Q_{\mathrm{f}, \text { in }}=\int_{H-h_{4}}^{H} u_{\mathrm{f}} \mathrm{d} y \quad$ at $\quad x=0$,

$p_{\mathrm{l}}=P_{\mathrm{dwn}}, \quad p_{\mathrm{f}}=0 \quad$ at $\quad x=1$.

For the leading-order system to reduce in a similar manner to the single-cell-population model of Pearson et al. (2014), we must show that the cell volume fractions $\theta_{\mathrm{u}}$ and $\theta_{\mathrm{d}}$ are independent of $y$. Given that $\partial p_{\mathrm{w}} / \partial y=0$ from $(3.19 b)$, the leading-order cell phase $y$-momentum equations (3.17) can be put into the following matrix form:

$\mathbf{A}\left(\begin{array}{c}\frac{\partial \theta_{\mathrm{u}}}{\partial y} \\ \frac{\partial \theta_{\mathrm{d}}}{\partial y}\end{array}\right)=\mathbf{0}$

where

$\mathbf{A}=\left(\begin{array}{cc}-\Pi_{\mathrm{u}}-\theta_{\mathrm{u}} \frac{\partial \Pi_{\mathrm{u}}}{\partial \theta_{\iota}}+\psi_{\mathrm{ns}} \theta_{\mathrm{s}} & -\theta_{\mathrm{u}} \frac{\partial \Pi_{\mathrm{u}}}{\partial \theta_{\mathrm{d}}} \\ -\theta_{\mathrm{d}} \frac{\partial \Pi_{\mathrm{d}}}{\partial \theta_{\mathrm{u}}} & -\Pi_{\mathrm{d}}-\theta_{\mathrm{d}} \frac{\partial \partial \Pi_{\mathrm{d}}}{\partial \theta_{\mathrm{d}}}+\psi_{\mathrm{ns}} \theta_{\mathrm{s}}\end{array}\right)$.

In general, the determinant of the matrix in (3.29) will be non-zero, i.e.

$$
\begin{array}{r}
\left(-\Pi_{\mathrm{u}}-\theta_{\mathrm{u}} \frac{\partial \Pi_{\mathrm{u}}}{\partial \theta_{\mathrm{u}}}+\psi_{\mathrm{ns}} \theta_{\mathrm{s}}\right)\left(-\Pi_{\mathrm{d}}-\theta_{\mathrm{d}} \frac{\partial \Pi_{\mathrm{d}}}{\partial \theta_{\mathrm{d}}}+\psi_{\mathrm{ns}} \theta_{\mathrm{s}}\right) \\
-\theta_{\mathrm{u}} \theta_{\mathrm{d}} \frac{\partial \Pi_{\mathrm{u}}}{\partial \theta_{\mathrm{d}}} \frac{\partial \Pi_{\mathrm{d}}}{\partial \theta_{\mathrm{u}}} \neq 0, \quad(3.31)
\end{array}
$$

from which we can conclude that we must have $\partial \theta_{\mathrm{u}} / \partial y=$ $\partial \theta_{\mathrm{d}} / \partial y=0$. Hence, similarly to the single-cell-population models, we have $\theta_{i}=\theta_{i}(x, t)(i=\mathrm{u}, \mathrm{d})$. We also find that the solute equations and boundary conditions yield

$c_{i}:=c(x, t) \quad(i=1, \mathrm{~m}, \mathrm{w}, \mathrm{f})$, for some $c(x, t)$ to be determined.

The remaining leading-order variables can be found in terms of the cell volume fractions $\theta_{\mathrm{u}}$ and $\theta_{\mathrm{d}}$, and the solute concentration $c$. In the lumen we have

$$
\begin{gathered}
p_{\mathrm{l}}=3 Q_{1, \mathrm{in}}(1-x)+P_{\mathrm{dwn}}, \\
u_{\mathrm{l}}=\frac{3}{2} Q_{\mathrm{l}, \mathrm{in}}\left(1-y^{2}\right), \quad v_{\mathrm{l}} \equiv 0,
\end{gathered}
$$

and in the membrane

$$
\begin{array}{r}
p_{\mathrm{m}}=\frac{1}{h_{2}}\left[\left(\frac{12 Q_{\mathrm{f}, \mathrm{in}}}{h_{4}^{3}}-3 Q_{1, \text { in }}\right)(1-x)-P_{\mathrm{dwn}}\right](y-1) \\
+3 Q_{1, \text { in }}(1-x)+P_{\mathrm{dwn}}, \\
u_{\mathrm{m}} \equiv 0, \\
v_{\mathrm{m}}=-\frac{\kappa_{\mathrm{m}}}{h_{2}}\left[\left(\frac{12 Q_{\mathrm{f}, \mathrm{in}}}{h_{4}^{3}}-3 Q_{1, \mathrm{in}}\right)(1-x)-P_{\mathrm{dwn}}\right] .
\end{array}
$$

In the cell layer, we consider the leading-order $x$ momentum equations for each cell phase,

$$
\begin{aligned}
-\theta_{i} & \frac{\partial p_{\mathrm{w}}}{\partial x}-\frac{\partial}{\partial x}\left(\theta_{i} \Pi_{i}\right)+\theta_{i} \frac{\partial^{2} u_{i}}{\partial y^{2}} \\
& +\psi_{\mathrm{ns}} \theta_{\mathrm{s}} \frac{\partial \theta_{i}}{\partial x}-\zeta_{\mathrm{ns}} \theta_{i} \theta_{\mathrm{s}} u_{i}=0 \quad(i=\mathrm{u}, \mathrm{d}) .
\end{aligned}
$$

These can be solved, along with (3.24a) and (3.25a), to find

$$
\begin{array}{r}
u_{i}=\frac{\widetilde{M}_{i}(x, t)}{\zeta_{\mathrm{ns}} \theta_{\mathrm{s}}}\left\{\frac{\cosh \left[\sqrt{\zeta_{\mathrm{ns}} \theta_{\mathrm{s}}}\left(H-h_{4}-y\right)\right]}{\cosh \left(\sqrt{\zeta_{\mathrm{ns}} \theta_{\mathrm{s}}} h_{3}\right)}-1\right\} \\
(i=\mathrm{u}, \mathrm{d}),
\end{array}
$$

where

$$
\begin{gathered}
\widetilde{M}_{i}:=\frac{\partial p_{\mathrm{w}}}{\partial x}+\frac{1}{\theta_{i}} \tilde{\Phi}_{i}\left(\theta_{i}, \theta_{j}\right) \frac{\partial \theta_{i}}{\partial x}+\frac{\partial \Pi_{i}}{\partial \theta_{j}} \frac{\partial \theta_{j}}{\partial x}, \\
\tilde{\Phi}_{i}:=\Pi_{i}+\theta_{i} \frac{\partial \Pi_{i}}{\partial \theta_{i}}-\psi_{\mathrm{ns}} \theta_{\mathrm{s}} \quad(i, j=\mathrm{u}, \mathrm{d}, i \neq j) .
\end{gathered}
$$

The remaining cell layer variables are

$p_{\mathrm{w}}=\frac{12 Q_{\mathrm{f}, \mathrm{in}}}{h_{4}^{3}}(1-x), \quad u_{\mathrm{w}}=\frac{12 Q_{\mathrm{f}, \text { in }}}{h_{4}^{3} \theta_{\mathrm{s}} \zeta_{\mathrm{ws}}}$,

with $v_{\mathrm{u}}$ and $v_{\mathrm{d}}$ given implicitly by their respective mass equations in (3.15), and $v_{\mathrm{w}}$ by (3.18). Finally, in the upper fluid layer we find

$p_{\mathrm{f}}=\frac{12 Q_{\mathrm{f}, \text { in }}}{h_{4}^{3}}(1-x)$,

$u_{\mathrm{f}}=-\frac{6 Q_{\mathrm{f}, \mathrm{in}}}{h_{4}^{3}}\left[y^{2}+\left(h_{4}-2 H\right)(y-H)-H^{2}\right]$,

$v_{\mathrm{f}} \equiv 0$.

We must now determine an equation for the leadingorder concentration $c$, and do so through consideration 
of the solute equations at $\mathrm{O}(\varepsilon)$. This differs from the analysis of the single-cell-population models in Pearson et al. (2013) and Pearson et al. (2014) in which we had to go to $\mathrm{O}\left(\varepsilon^{2}\right)$; this is due to the increased size of $\varepsilon^{2} \mathrm{Pe}$ here compared to our previous models. At $\mathrm{O}(\varepsilon)$ we have (briefly returning to subscript 0,1 notation for clarity)

$$
\begin{gathered}
\varepsilon \operatorname{Pe} u_{\mathrm{l}_{0}} \frac{\partial c}{\partial x}=\frac{\partial^{2} c_{\mathrm{l}_{1}}}{\partial y^{2}}, \quad \frac{\partial^{2} c_{\mathrm{m}_{1}}}{\partial y^{2}}=0, \\
\theta_{\mathrm{w}_{0}} \frac{\partial^{2} c_{\mathrm{w}_{1}}}{\partial y^{2}}=-\mathcal{R}, \quad \varepsilon \operatorname{Pe} u_{\mathrm{f}_{0}} \frac{\partial c}{\partial x}=\frac{\partial^{2} c_{\mathrm{f}_{1}}}{\partial y^{2}},
\end{gathered}
$$

together with the corresponding continuity of solute and of solute flux conditions on the interfaces between layers, and no flux of solute conditions on the lumen centreline and bioreactor top. Integrating the equations in (3.44) across their respective layers (appropriately weighted in the membrane and cell layer) and adding the resulting equations yields the following equation for $c$ :

$\varepsilon \operatorname{Pe}\left(Q_{1, \text { in }}+Q_{\mathrm{f}, \text { in }}\right) \frac{\partial c}{\partial x}=h_{3} \mathcal{R}\left(\theta_{\mathrm{u}}, \theta_{\mathrm{d}}\right)$.

This couples to the equations for the remaining unknowns $\theta_{\mathrm{u}}$ and $\theta_{\mathrm{d}}$, obtained by integrating the mass equations in (3.15) across the cell layer:

$\frac{\partial \theta_{i}}{\partial t}+\frac{\partial Q_{i}}{\partial x}=J_{i}\left(\theta_{\mathrm{u}}, \theta_{\mathrm{d}}, c\right) \quad(i=\mathrm{u}, \mathrm{d})$

where

$$
\begin{gathered}
Q_{i}=\frac{\widetilde{M}_{i}(x, t)}{\zeta_{\mathrm{ns}} \theta_{\mathrm{s}}}\left(\frac{\tanh (\alpha)}{\alpha}-1\right) \theta_{i} \quad(i=\mathrm{u}, \mathrm{d}), \\
\alpha:=\sqrt{\zeta_{\mathrm{ns}} \theta_{\mathrm{s}}} h_{3},
\end{gathered}
$$

and $\widetilde{M}_{i}(i=\mathrm{u}, \mathrm{d})$ are defined in (3.39).

We must finally prescribe the remaining up- and downstream conditions which are needed in order to close the system. We prescribe an inlet concentration and also impose no flux of cells out of the cell layer, so that we have

$$
\begin{aligned}
Q_{i} & =0 \quad(i=\mathrm{u}, \mathrm{d}) & \text { at } & x=0,1, \\
c & =c_{\mathrm{in}} & \text { at } & x=0 .
\end{aligned}
$$

\subsection{Summary of the reduced model}

In summary, we have reduced the 4-layer, two-cellpopulation model to a coupled system of three PDEs for the leading-order cell volume fractions $\theta_{\mathrm{u}}$ and $\theta_{\mathrm{d}}$ and solute concentration $c$. To recap, we have

$$
\begin{aligned}
& \frac{\partial \theta_{i}}{\partial t}+\frac{\partial Q_{i}}{\partial x}=J_{i}\left(\theta_{\mathrm{u}}, \theta_{\mathrm{d}}, c\right) \quad(i=\mathrm{u}, \mathrm{d}), \\
& \varepsilon \operatorname{Pe}\left(Q_{1, \text { in }}+Q_{\mathrm{f}, \text { in }}\right) \frac{\partial c}{\partial x}=h_{3} \mathcal{R}\left(\theta_{\mathrm{u}}, \theta_{\mathrm{d}}\right),
\end{aligned}
$$

subject to

$$
\begin{aligned}
Q_{i} & =0 \quad(i=\mathrm{u}, \mathrm{d}) & \text { at } & x=0,1, \\
c & =c_{\text {in }} & \text { at } & x=0,
\end{aligned}
$$

and where $Q_{i}(i=\mathrm{u}, \mathrm{d})$ are given in (3.47).

\subsection{Reduced 3-layer model}

If only three layers are considered, we must solve for $p_{\mathrm{w}}$ as well as $\theta_{\mathrm{u}}, \theta_{\mathrm{d}}$, and $c$ (see Pearson et al. (2014)). The analysis follows through in a similar manner (as in $\S 3.3$, going to $\mathrm{O}(\varepsilon)$ for the solute equation instead of $\mathrm{O}\left(\varepsilon^{2}\right)$ ), and the resulting coupled system of equations is

$$
\begin{gathered}
\frac{\partial \theta_{i}}{\partial t}+\frac{\partial Q_{i}}{\partial x}=J_{i} \quad(i=\mathrm{u}, \mathrm{d}) \\
\varepsilon \operatorname{Pe} Q_{1, \text { in }} \frac{\partial c}{\partial x}=h_{3} \mathcal{R}, \\
\frac{\partial}{\partial x}\left(Q_{\mathrm{u}}+Q_{\mathrm{d}}+Q_{\mathrm{w}}\right)=-\frac{\kappa_{\mathrm{m}} \phi_{\mathrm{m}}}{h_{2} h_{3}}\left[p_{\mathrm{w}}\right. \\
\left.-3 Q_{1, \text { in }}(1-x)-P_{\mathrm{dwn}}\right],
\end{gathered}
$$

where

$$
\begin{aligned}
Q_{i} & =\frac{\widetilde{M}_{i}(x, t) \theta_{i}}{\zeta_{\mathrm{ns}} \theta_{\mathrm{s}} \alpha \sinh (\alpha)}[2 \cosh (\alpha)-\alpha \sinh (\alpha)-2] \\
\qquad(i=\mathrm{u}, \mathrm{d}), \quad \alpha:=\sqrt{\zeta_{\mathrm{ns}} \theta_{\mathrm{s}}} h_{3}, & \\
Q_{\mathrm{w}} & =-\frac{\theta_{\mathrm{w}}}{\zeta_{\mathrm{ws}} \theta_{\mathrm{s}}} \frac{\partial p_{\mathrm{w}}}{\partial x}
\end{aligned}
$$

subject to the boundary conditions

$$
\begin{array}{ll}
Q_{i}=0 \quad(i=\mathrm{u}, \mathrm{d}) \quad \text { at } \quad x=0,1, \\
Q_{\mathrm{w}}=\frac{Q_{\mathrm{e}, \mathrm{in}}}{h_{3}}, \quad c=c_{\mathrm{in}} & \text { at } \quad x=0, \\
p_{\mathrm{w}}=0 & \text { at } \quad x=1,
\end{array}
$$

where $\widetilde{M}_{i}(i=\mathrm{u}, \mathrm{d})$ is defined in $(3.39)$.

\section{Numerical results}

Numerical results were obtained using the method of lines, first discretising in $x$ and then performing the time integration using the MATLAB function ode15s. As equations $(3.50 b)$ and $(3.53 b)$ only involve an advection term for the solute concentration, a regularising diffusion term was included for the purposes of numerical solution. The coefficient of this term was taken to be $\mathrm{O}\left(10^{-4}\right)$ for all results presented here, and it was verified that reduction of this coefficient to $\mathrm{O}\left(10^{-5}\right)$ had no significant impact upon results. Due to a lack of experimental data for initial cell volume fractions, all results were obtained with the initial conditions $\theta_{\mathrm{u}}=0.3$ and 
$\theta_{\mathrm{d}}=0$, and we note that the value of $\theta_{\mathrm{u}}$ would need to be verified in order to obtain quantitative predictions. We also set the pseudo-initial conditions $c(x, 0)=$ $c_{\mathrm{in}}, p_{\mathrm{w}}(x, 0)=1$ where necessary.

In the following sections we present results for two different case studies, each relating to a specific constitutive choice for $J_{\mathrm{u}}$ and $J_{\mathrm{d}}$, motivated by experimental observations of cell differentiation stimuli. In both cases we assume that the two cell populations take up the growth factor at the same rate, so that the constitutive form for $\mathcal{R}$ is given by

$\mathcal{R}=-\Gamma_{\mathrm{R} 1} c \theta_{\mathrm{w}} \theta_{\mathrm{u}}-\Gamma_{\mathrm{R} 1} c \theta_{\mathrm{w}} \theta_{\mathrm{d}}=-\Gamma_{\mathrm{R} 1} c \theta_{\mathrm{w}}\left(\theta_{\mathrm{u}}+\theta_{\mathrm{d}}\right)$,

similar to that used for solute uptake in the chemoattractant case studies in Pearson et al. (2013).

\subsection{Optimisation parameters}

As discussed in $\S 1$, minimising the necessary quantity of growth factor experimentally is of particular importance due to their high cost. As an example to illustrate how our model may be used in this way, for each case study we discuss experimental operating conditions (inlet fluid fluxes, inlet concentration, and experiment end time $\tilde{t})$ which result in a minimum percentage $(98 \%$, following our discussion in §3.1) of differentiated cells being obtained, whilst also taking into account the yield of differentiated cells and amount of growth factor used. Once validated, similar analysis of our model could be used to make more quantitative predictions and inform experimental protocol.

We denote the percentage of cells which are of the differentiated phenotype at time $t$ by $\pi_{\mathrm{d}}(t)$, and the yield of differentiated cells by $\mu_{\mathrm{d}}(t)$. These are defined, respectively, by

$\pi_{\mathrm{d}}(t):=\frac{100 \mu_{\mathrm{d}}(t)}{\mu_{\mathrm{u}}(t)+\mu_{\mathrm{d}}(t)}$,

and

$\mu_{i}(t):=\int_{0}^{1} \theta_{i}(x, t) \mathrm{d} x \quad(i=\mathrm{u}, \mathrm{d})$.

We also estimate the quantity of growth factor $q_{\text {in }}$ supplied to the system by the leading-order flux of solute that has entered the system at time $t$. In the 3-layer model this is

$$
\begin{aligned}
q_{\text {in }}(t) & :=\left.\varepsilon \operatorname{Pe} \int_{0}^{t} \int_{0}^{1} c\left(x, t^{\prime}\right) u_{1}\left(y, t^{\prime}\right)\right|_{x=0} \mathrm{~d} y \mathrm{~d} t^{\prime} \\
& =\varepsilon \operatorname{Pe} Q_{1, \text { in }} t c_{\text {in }},
\end{aligned}
$$

and in the 4-layer model

$$
\begin{aligned}
q_{\text {in }}(t):=\varepsilon \operatorname{Pe} \int_{0}^{t}\left(\left.\int_{0}^{1} c\left(x, t^{\prime}\right) u_{\mathrm{l}}\left(y, t^{\prime}\right)\right|_{x=0} \mathrm{~d} y\right. \\
\left.\quad+\left.\int_{H-h_{4}}^{H} c\left(x, t^{\prime}\right) u_{\mathrm{f}}\left(y, t^{\prime}\right)\right|_{x=0} \mathrm{~d} y\right) \mathrm{d} t^{\prime} \\
=\varepsilon \operatorname{Pe}\left(Q_{\mathrm{l}, \text { in }}+Q_{\mathrm{f}, \text { in }}\right) t c_{\mathrm{in}} .
\end{aligned}
$$

Finally, we calculate the amount of growth factor taken up by the cells by time $t, q_{\text {up }}$. For both the 3 - and 4layer models this is given by

$q_{\text {up }}(t):=-h_{3} \int_{0}^{t} \int_{0}^{1} \mathcal{R}\left(x, t^{\prime}\right) \mathrm{d} x \mathrm{~d} t^{\prime}$,

where we note that the minus sign results in a positive value for $q_{\text {up }}$. The quantities $q_{\text {in }}(t)$ and $q_{\text {up }}(t)$ give estimates for the total amount of growth factor supplied during the experiment up to time $t$ (assuming the culture medium is not recycled during the experiment) and the amount of growth factor 'lost' during the experiment up to time $t$, respectively. This provides a measure of how expensive each set of operating conditions are likely to be based on the amount of growth factor required, assuming that any not taken up by the cells can be retained and used later. Practically, however, only around $95 \%$ of any unused growth factor can be recovered $^{2}$ and so $q_{\text {up }}$ should be interpreted as an underestimate. We estimate the integrals in (4.2)-(4.6) from our numerical solutions using the MATLAB function trapz.

\subsection{Growth factor-dependent differentiation}

In the first case study, we consider cell differentiation which is enhanced solely by the growth factor concentration. Several examples of this type of relationship can be found in the experimental literature, in particular relating to osteogenesis (Midy and Plouet 1994; Mayer et al. 2005) and endothelial differentiation (Breier et al. 1992; Peters et al. 1993; Mayer et al. 2005). We take the following forms for the mass transfer functions:

$J_{\mathrm{u}}=\Gamma_{\mathrm{nw}} \theta_{\mathrm{u}} \theta_{\mathrm{w}}-\Gamma_{\mathrm{ud}} \theta_{\mathrm{u}} \theta_{\mathrm{w}} \frac{c}{K+c}-\Gamma_{\mathrm{wn}} \theta_{\mathrm{u}}$,
$J_{\mathrm{d}}=\Gamma_{\mathrm{nw}} \theta_{\mathrm{d}} \theta_{\mathrm{w}}+\Gamma_{\mathrm{ud}} \theta_{\mathrm{u}} \theta_{\mathrm{w}} \frac{c}{K+c}-\Gamma_{\mathrm{wn}} \theta_{\mathrm{d}}$.

In the above, the first and last terms in each of $J_{\mathrm{u}}$ and $J_{\mathrm{d}}$ are the same as the cell proliferation and death terms in Pearson et al. (2013) in which nutrient is assumed to be in abundance. The middle terms in each constitutive law represent the differentiation of the undifferentiated to the differentiated cell population, with 
(a)

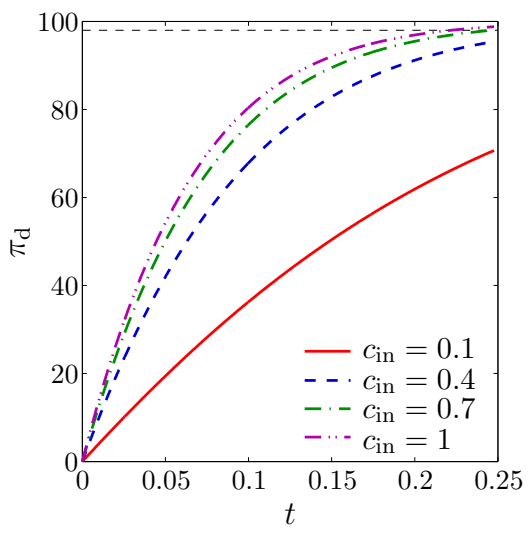

(b)

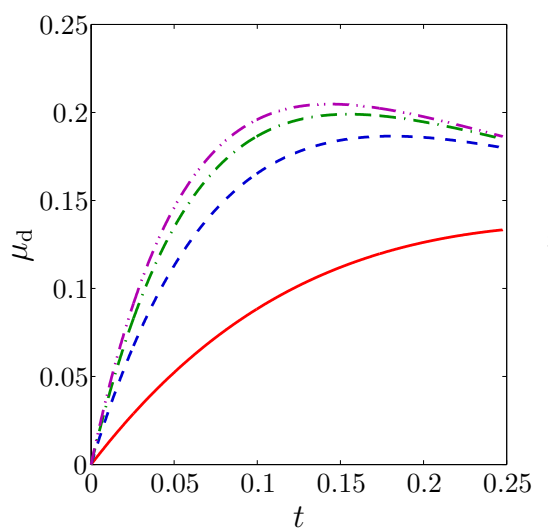

(c)

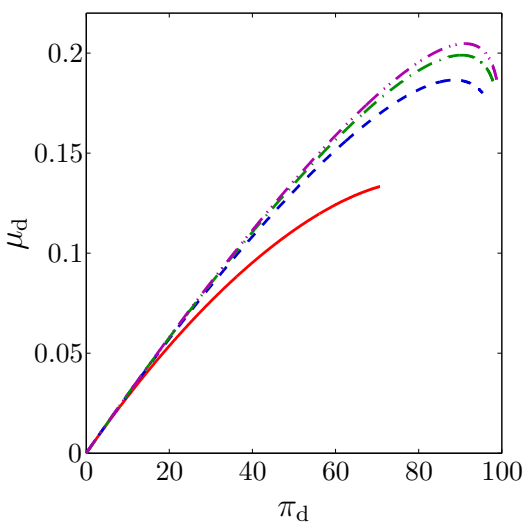

Fig. 3 Results for the growth factor-dependent differentiation case study. (a) Percentage of differentiated cells $\pi_{\mathrm{d}}$ versus $t$, with the horizontal dashed black line denoting $\pi_{\mathrm{d}}=98$; (b) differentiated cell yield $\mu_{\mathrm{d}}$ versus $t$; and (c) a direct comparison $\pi_{\mathrm{d}}$ versus $\mu_{\mathrm{d}}$, for the 3-layer model and varying inlet concentration values $c_{\mathrm{in}}$. The legend in (a) applies to all subfigures. The lumen inlet flux was fixed at $Q_{1, \text { in }}=1$ and all other parameter values take their fixed values from Table 3

a constant differentiation rate coefficient $\Gamma_{\mathrm{ud}}$. The differentiation rate is assumed proportional to the volume fraction of undifferentiated cells so that, in particular, no differentiation occurs in the absence of undifferentiated cells. Finally, the dependence of cell differentiation rate on the growth factor concentration is included via a Michaelis-Menten type term.

Results for this case study, in which the inlet concentration $c_{\text {in }}$ is varied, can be seen in Figure 3. Only results from the 3-layer model are plotted, as in this case the 4-layer model results are qualitatively similar (not shown). This suggests that, for this case study, solving the less computationally intensive 4-layer model is sufficient to determine the behaviour of either system in response to variations in $c_{\text {in }}$. Figure $3(\mathrm{a})$ shows that the differentiated cell percentage $\pi_{\mathrm{d}}$ increases over time for all $c_{\text {in }}$ values, with a more rapid initial increase and greater final value for higher values of the inlet concentration $c_{\mathrm{in}}$. This is to be expected given the dependence of cell differentiation on $c$ in (4.7) and (4.8). Furthermore, the decreasing difference between subsequent $\pi_{\mathrm{d}}$ plots as $c_{\text {in }}$ increases shows that, at higher inlet concentrations, the differentiation term is approaching its saturating value, i.e. $\Gamma_{\mathrm{ud}} \theta_{\mathrm{u}} \theta_{\mathrm{w}}$.

However, for all $c_{\text {in }}$ values, the differentiated cell yield $\mu_{\mathrm{d}}$ reaches a peak before decreasing towards the end of the experiment due to cell death (see Figure $3(\mathrm{~b})$, where we note that this behaviour for $c_{\text {in }}=0.1$ is out of the range of $t$ considered). The peak value is greater, and achieved at earlier times, as $c_{\text {in }}$ increases, behaviours which can be attributed to two factors. First of all, for higher $c_{\text {in }}$ values the rate of differentiation will be greater, and so $\mu_{\mathrm{d}}$ will increase more quickly and to a higher value. Secondly, a higher differentiated cell yield also results in a higher cell death rate (see equation (4.8)), and so its effect in decreasing $\mu_{\mathrm{d}}$ becomes apparent at earlier times. Figure 3(c) shows a plot of $\pi_{\mathrm{d}}$ versus $\mu_{\mathrm{d}}$ which clearly shows the trends discussed above: as $\pi_{\mathrm{d}}$ increases over time, at a particular point the maximum yield is reached and thereafter $\mu_{\mathrm{d}}$ decreases.

From the three plots in Figure 3 we can see that the required value of $\pi_{\mathrm{d}}=98$ is only reached within the timeframe considered (which we recall corresponds to the maximum experimental end time of 4 weeks) for $c_{\mathrm{in}}=0.7$ and 1 , with corresponding values of $\mu_{\mathrm{d}}=$ 0.185 and 0.193 , and at experimental end times $\tilde{t}=$ 0.247 and 0.222 , respectively. The resulting quantities of growth factor supplied and used are $q_{\text {in }}(\tilde{t})=0.885$ and 1.138 , and $q_{\text {up }}(\tilde{t})=0.043$ and 0.056 , respectively. Thus, although setting $c_{\mathrm{in}}=1$ reaches the required value of $\pi_{\mathrm{d}}$ earlier, and with a $4 \%$ higher yield of cells, compared with $c_{\text {in }}=0.7$, a larger quantity of growth factor is needed and more is also used overall (in fact $29 \%$ and $30 \%$ more, respectively). In this case, therefore, a compromise must be made between the quantity of growth factor and yield of cells.

We note that in this case study, changing the inlet fluid flux values will affect the distribution of growth factor and therefore also $\pi_{\mathrm{d}}$ and $\mu_{\mathrm{d}}$. However, trends were shown to be qualitatively similar to those obtained in the next case study, and we therefore postpone discussion of varying fluid fluxes until $§ 4.3$. 
Table 4 Values of the time $\tilde{t}$, differentiated cell yield $\mu_{\mathrm{d}}$, supplied growth factor $q_{\text {in }}$ and used growth factor $q_{\text {up }}$ corresponding to the point at which the percentage of differentiated cells $\pi_{\mathrm{d}}$ reaches 98 for the combined case study, for the 3-layer $\left(Q_{1, \text { in }}\right.$ varying) and 4-layer $\left(Q_{1, \text { in }}\right)$ models

\begin{tabular}{llllll}
\hline Model & Inlet fluid flux and value & $\tilde{t}$ & $\mu_{\mathrm{d}}(\tilde{t})$ & $q_{\text {in }}(\tilde{t})$ & $q_{\text {up }}(\tilde{t})$ \\
\hline 3-layer & $Q_{1, \text { in }}=1$ & 0.1944 & 0.2017 & 0.9958 & 0.049 \\
3-layer & $Q_{1, \text { in }}=1.5$ & 0.1635 & 0.2126 & 1.2563 & 0.0419 \\
3-layer & $Q_{1, \text { in }}=2$ & 0.1533 & 0.2163 & 1.5706 & 0.0395 \\
3-layer & $Q_{1, \text { in }}=2.5$ & 0.1565 & 0.2149 & 2.0042 & 0.0403 \\
\hline 4-layer & $Q_{\mathrm{f} \text {,in }}=0.5$ & 0.1273 & 0.2268 & 0.974 & 0.0217 \\
4-layer & $Q_{\mathrm{f}, \text { in }}=1$ & 0.1372 & 0.2219 & 1.3997 & 0.0289 \\
\hline
\end{tabular}

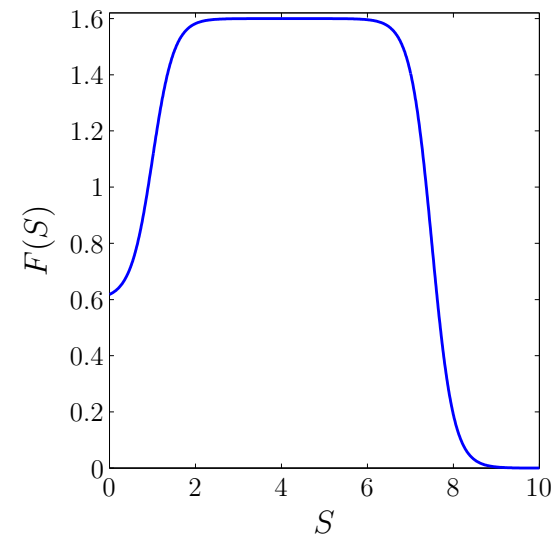

Fig. 4 Plot of $F(S)$ versus the dimensionless shear stress $S$, within the range of values achieved by our model

4.3 Growth factor- and shear stress-dependent differentiation

Next we consider the case where cell differentiation is enhanced both by growth factor concentration and fluid shear stress, as observed by Wu et al. (2008) for endothelial cell differentiation. We take the same approximation for the fluid shear stress $S$ as in Pearson et al. (2014), that is,

$S=\frac{\left|\frac{\partial p_{\mathrm{w}}}{\partial x}\right|}{\theta_{\mathrm{w}}}$.

In calculating $S$ for the 3-layer setup in which we do not have an explicit expression for $p_{\mathrm{w}}$, we estimate the pressure gradient $\partial p_{\mathrm{w}} / \partial x$ from the numerical solution to $p_{\mathrm{w}}$ using central differences. The constitutive forms for $J_{\mathrm{u}}$ and $J_{\mathrm{d}}$ in this case are

$$
\begin{aligned}
& J_{\mathrm{u}}=\Gamma_{\mathrm{nw}} \theta_{\mathrm{u}} \theta_{\mathrm{w}}-\Gamma_{\mathrm{ud}} \theta_{\mathrm{u}} F(S) \theta_{\mathrm{w}} \frac{c}{K+c}-\Gamma_{\mathrm{wn}} \theta_{\mathrm{u}}, \\
& J_{\mathrm{d}}=\Gamma_{\mathrm{nw}} \theta_{\mathrm{d}} \theta_{\mathrm{w}}+\Gamma_{\mathrm{ud}} \theta_{\mathrm{u}} F(S) \theta_{\mathrm{w}} \frac{c}{K+c}-\Gamma_{\mathrm{wn}} \theta_{\mathrm{d}},
\end{aligned}
$$

where the cell proliferation and death terms are unchanged from $\S 4.2$, and $F(S)$ has been added to the cell differentiation term to represent the functional dependence of the cell differentiation on the shear stress. Given that shear stress has been observed to increase cell differentiation only once a certain physiological threshold of shear stress is reached (Wu et al. 2008), the form we choose for $F(S)$ is similar to that used in Pearson et al. (2014):

$F(S)=0.3+0.5 \tanh (2 S-2)-0.8 \tanh (2 S-15)$.

The coefficients in (4.12) have been chosen to capture the possible behaviours of the cell population within the range of computed shear stresses in our model, and we assume that if high enough levels of shear stress are exceeded, then differentiation is no longer promoted (this assumption is motivated by the experimental observation that high shear stress can be detrimental to cells Park et al. (2007); Keane et al. (2003); Tourovskaia et al. (2004); Korin et al. (2009)). A plot of $F(S)$ can be seen in Figure 4.

In this case study we consider the effect of varying both the inlet growth factor concentration and inlet fluid fluxes on differentiated cell percentage, with results shown in Figures 5-7. Firstly, in Figure 5 the inlet concentration $c_{\text {in }}$ is varied for two different values of $Q_{1, \text { in }}$ and results plotted for both the 3- and 4-layer models, showing qualitatively similar trends to results from $\S 4.2$. Figures $5(\mathrm{a})$ and (b) show that for the lower value of $Q_{1, \text { in }}=0.05, \pi_{\mathrm{d}}$, and $\mu_{\mathrm{d}}$ are always lower in the 3-layer model than in the 4-layer model, whereas the opposite is true for the higher lumen flux of $Q_{1, \text { in }}=1$ 
(a)

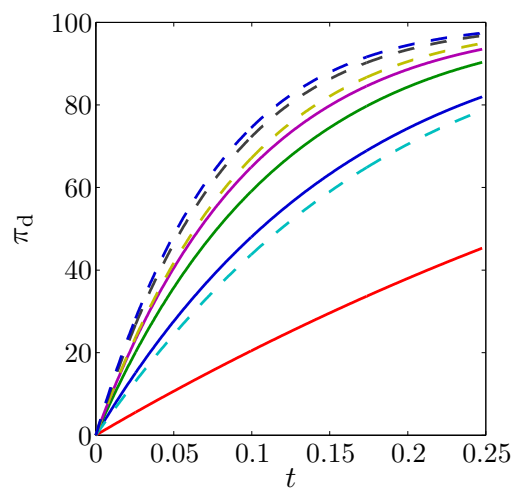

(c)

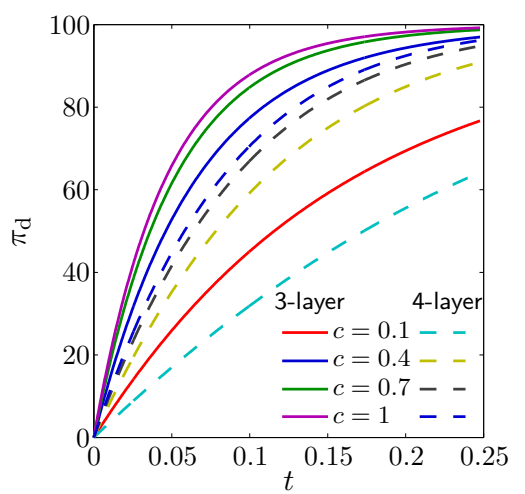

(b)

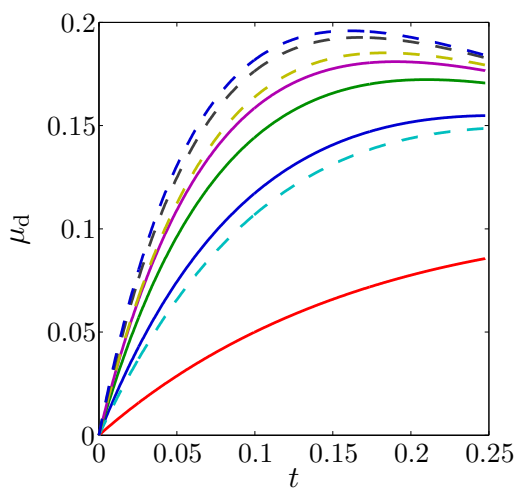

(d)

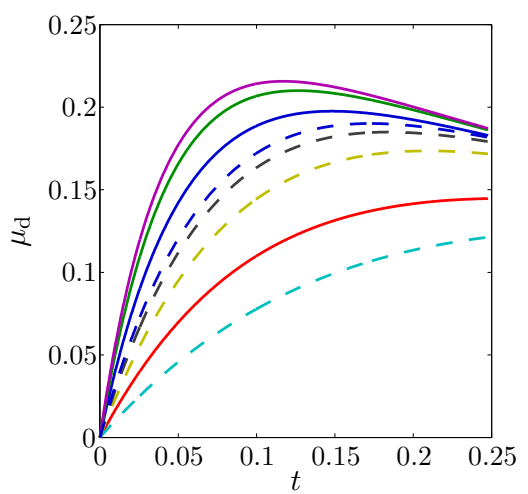

Fig. 5 Plots of $\pi_{\mathrm{d}}$ and $\mu_{\mathrm{d}}$ versus $t$ for varying values of the inlet growth factor concentration $c_{\text {in }}$ in the combined growth factor- and shear stress-dependent differentiation case study, for the 3- and 4-layer models. In (a), (b) $Q_{1, \text { in }}=0.05$, and in (c), (d) $Q_{1, \text { in }}=1$. The legend in (c) applies to all subfigures. The upper fluid layer inlet flux and cell layer height (for the 4-layer model) were fixed at $Q_{\mathrm{f} \text {,in }}=0.1$ and $h_{3}=1$ respectively. All other parameter values take their fixed values from Table 3

(see Figures 5(c) and (d)). This is due to the dependence of the shear stress on the lumen inlet fluid flux, which is greater in the 3-layer model compared with the 4-layer model (where its only influence is through $\theta_{\mathrm{w}}$ ). For $Q_{1, \text { in }}=0.05, S$ (and so $F(S)$ ) in the 3-layer model is on average lower than that in the 4-layer model, so that differentiation is promoted more in the latter, and vice versa at the higher fluid flux value (results not shown). These plots demonstrate that, with the additional shear stress dependence, there is a greater difference between the 3 - and 4-layer model results compared to $§ 4.2$.

The dependence of $\pi_{\mathrm{d}}$ and $\mu_{\mathrm{d}}$ on the lumen inlet fluid flux is shown in Figure 6. Changing $Q_{1, \text { in }}$ has very little effect on $\pi_{\mathrm{d}}$ or $\mu_{\mathrm{d}}$ for the 4-layer model, as $\theta_{\mathrm{u}}$, $\theta_{\mathrm{d}}$, and $c$ do not vary much with variations in $Q_{1, \text { in }}$ and the reduced water pressure $\partial p_{\mathrm{w}} / \partial x$ (which contributes to $S$ ) is independent of the lumen inlet fluid flux (see equation $(3.40 a)$ ). Plots of $\pi_{\mathrm{d}}$ and $\mu_{\mathrm{d}}$ are therefore only given for $Q_{1, \text { in }}=1$ but can be taken to be represen- tative of all lumen inlet flux values. For the 3-layer model, $\pi_{\mathrm{d}}$ increases more quickly and reaches a higher final value as $Q_{1, \text { in }}$ increases, until $Q_{1, \text { in }}=2$. For large enough times $t$, this trend is then reversed as $Q_{1, \text { in }}$ is increased above 2. This non-monotonic behaviour is also found in $\mu_{\mathrm{d}}$, with $Q_{1, \text { in }}=2$ achieving the maximum yield over the range of time considered $(0<t<T)$, as well as the peaked trends in time seen in $\S 4.2$. This nonmonotonicity is partly due to the effect of shear stress. As $Q_{1, \text { in }}$ increases from 2 to 5 , the value of $S$ near $x=1$ for the time range considered increases from around 3 to more than 10 (results not shown), and so $F(S)$ goes from the region in which differentiation is enhanced, into that at which the shear stress is too high and differentiation is no longer stimulated. However, similar nonmonotonic behaviour is observed in $\pi_{\mathrm{d}}$ and $\mu_{\mathrm{d}}$ if $Q_{1 \text {,in }}$ is varied in the case study from $\S 4.2$ in which the effects of shear stress on cell differentiation are not accounted for (results not shown), and so the effect cannot be due 
(a)

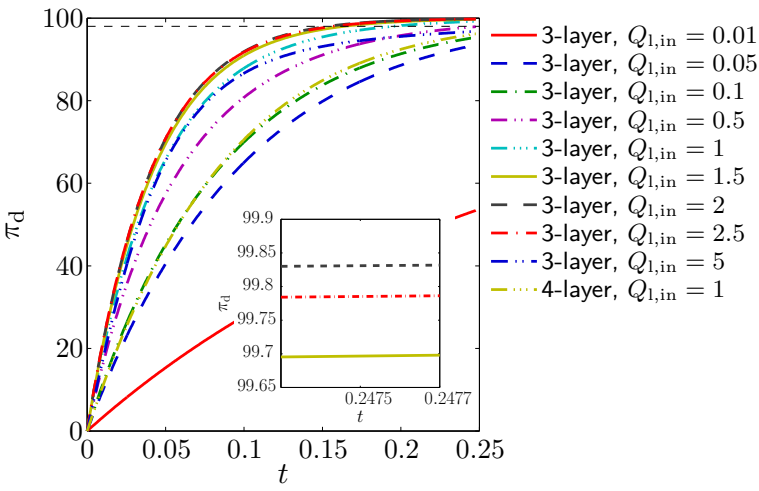

(b)

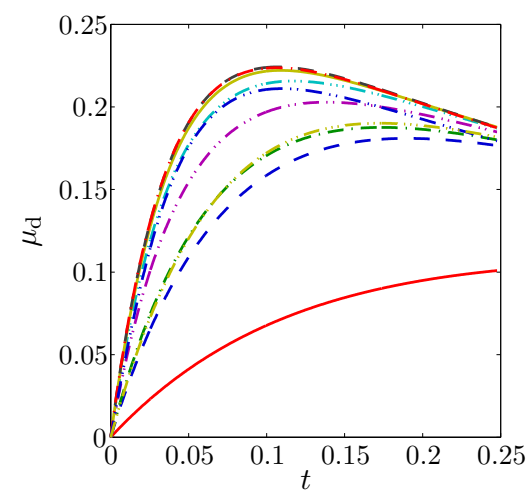

Fig. 6 Plot of (a) $\pi_{\mathrm{d}}$, and (b) $\mu_{\mathrm{d}}$, versus $t$ for varying values of the lumen inlet flux $Q_{1 \text {,in }}$, for both the 3 - and 4-layer models in the combined growth factor- and shear stress-dependent differentiation case study. The horizontal dashed black line in (a) denotes $\pi_{\mathrm{d}}=98$, and the inset shows the detail of the monotonicity arising when $Q_{\text {lin }}$ is increased beyond 2 . The legend in (a) applies to both subfigures. The inlet concentration was fixed at $c_{\text {in }}=1$, and (for the 4-layer model) the upper fluid layer inlet flux $Q_{\mathrm{f}, \text { in }}=0.1$ and cell layer height $h_{3}=1$. All other parameter values take their fixed values from Table 3

(a)

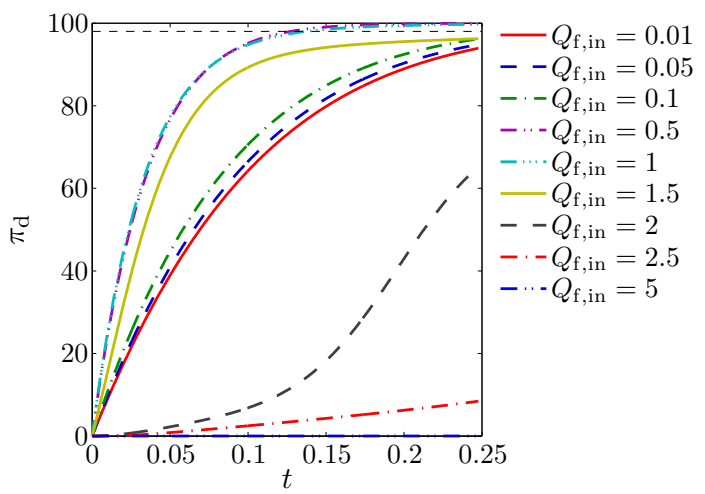

(b)

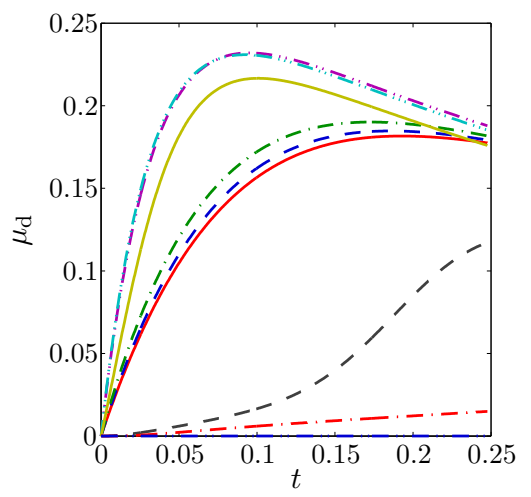

Fig. 7 Plot of (a) $\pi_{\mathrm{d}}$, and (b) $\mu_{\mathrm{d}}$, versus $t$ for varying values of the upper fluid layer inlet flux $Q_{\mathrm{f}, \text { in }}$, in the combined growth factor- and shear stress-dependent differentiation case study and for the 4-layer model. The horizontal dashed black line in (a) denotes $\pi_{\mathrm{d}}=98$. The legend in (a) applies to both subfigures. The inlet concentration was fixed at $c_{\mathrm{in}}=1$, the lumen inlet flux $Q_{1, \text { in }}=1$, and the cell layer height $h_{3}=1$. All other parameter values take their fixed values from Table 3

to shear stress alone. For large enough values of $Q_{1, \text { in }}$, advection becomes strong enough to 'sweep' more cells downstream, and this results in local variations in the values of $\theta_{\mathrm{u}}$ and $\theta_{\mathrm{d}}$. These differences feed into the cell proliferation, differentiation, and death rates (in a nonlinear way for the first two), and it is this complex combination which also contributes to the non-monotonic trends in $\pi_{\mathrm{d}}$ and $\mu_{\mathrm{d}}$ discussed above. This hypothesis is supported by repeating the same simulations with either an increased cell-scaffold drag $\zeta_{\text {ns }}$ or cell-scaffold traction parameter $\eta$. In both cases we would expect advection to have a lesser effect on cell distribution (due to cells being 'swept' downstream less readily), and we find that the non-monotonic behaviour does not arise between $Q_{1, \text { in }}=2$ and 2.5, but only for higher values of $Q_{1, \text { in }}$ (results not shown).

As can be seen in Figure 6, $\pi_{\mathrm{d}}$ reaches $98 \%$ in the 3-layer model for $Q_{1, \text { in }}=1,1.5,2$, and 2.5. The times $\tilde{t}$ at which this value is achieved, along with the corresponding values of $\mu_{\mathrm{d}}, q_{\text {in }}$, and $q_{\text {up }}$ can be found in Table 4 , and show that $q_{\text {in }}$ increases with $Q_{1, \text { in }}$, whilst $\tilde{t}, \mu_{\mathrm{d}}$, and $q_{\text {up }}$ follow the non-monotonic dependence of $\pi_{\mathrm{d}}$, with their trends reversing between $Q_{1, \text { in }}=2$ and $Q_{1, \text { in }}=2.5$. Hence in this case setting $Q_{1 \text {,in }}$ to 2 results 
in both the highest cell yield and the lowest growth factor consumption. However, if growth factor was not easily recoverable in a particular experiment, it may be worth setting the lumen inlet fluid flux to a lower value to reduce the amount $q_{\text {in }}$ supplied to the system, with a slight compromise on cell yield. It is also worth noting here that both $q_{\text {in }}$ and $q_{\text {up }}$ are much more sensitive than $\mu_{\mathrm{d}}$ to changes in $Q_{1, \text { in }}$. For example, between $Q_{1, \text { in }}=1$ and 2 , there is a $19.4 \%$ decrease in $q_{\text {up }}$, and a $59.7 \%$ increase in $q_{\text {in }}$, but $\pi_{\mathrm{d}}$ only increases by $7.2 \%$. Thus it may not be worth increasing flow rates to achieve a very small gain in cell yield, if the corresponding cost is disproportionately higher.

Finally, in Figure 7 the dependence of $\pi_{\mathrm{d}}$ and $\mu_{\mathrm{d}}$ on $Q_{\mathrm{f}, \text { in }}$ can be seen for the 4-layer model. Here we also see the effect of the non-monotonic form for $F(S)$, as $\pi_{\mathrm{d}}$ increases more quickly and to a greater final value as $Q_{\mathrm{f}, \text { in }}$ increases up to 0.5 , but then the opposite trend is seen for higher fluid flux values. The differentiated cell yield follows a similar trend, with the greatest final value of $\mu_{\mathrm{d}}$ also achieved when $Q_{\mathrm{f} \text {,in }}=0.5$. In addition, for $Q_{\mathrm{f} \text {,in }}<2, \mu_{\mathrm{d}}$ reaches a peak value before the maximum end time $T=0.2477$, as seen in previous graphs. The shape of $\pi_{\mathrm{d}}$ over time changes once $Q_{\mathrm{f} \text {,in }}$ reaches 2 , increasing more slowly at first and then more sharply at later times; this is the opposite to the trend for lower fluid flux values. As $Q_{\mathrm{f} \text {,in }}$ increases above $2, \pi_{\mathrm{d}}$ increases approximately linearly with time but takes much lower values due to the higher values of $S$ obtained. From these results, it can be seen that $\pi_{\mathrm{d}}$ only reaches a value of 98 for $Q_{\mathrm{f}, \text { in }}=0.5$ and 1 , and hence it is not beneficial in this case to increase the upper fluid layer flux further. Corresponding values of $\tilde{t}, \mu_{\mathrm{d}}, q_{\mathrm{in}}$, and $q_{\mathrm{up}}$ can be found in Table 4 and show that here the best choice of operating condition is clear, as setting $Q_{\mathrm{f}, \text { in }}=0.5$ results in not only a higher final yield than $Q_{1, \text { in }}=1$, but also consumes less growth factor.

\section{Discussion}

We have formulated and solved a multiphase model for fluid flow, solute concentration, and the evolution of two cell populations, one undifferentiated and one differentiated, in a simplified HFMB setup. We have considered case studies in which cell differentiation is dependent either on the concentration of a growth factor, or on both growth factor concentration and fluid shear stress. Within these case studies, we varied the fluid fluxes into the lumen and upper fluid layer, as well as the inlet growth factor concentration, to determine their effect on the percentage and yield of differentiated cells within an experimental time frame. Furthermore, for a candidate required percentage of differentiated cells, we compared the corresponding differentiated cell yield, experiment end time, and quantity of growth factor used/required for different operating conditions, to illustrate their relative merits and how our model can be used to inform experimental procedure once validated. In each case, we identified ranges of the experimentally controllable parameter values within which the required percentage of differentiated cells was achieved. These ranges could be used as starting points for a more thorough parameter analysis to determine ideal operating conditions.

We have simplified the model formulation by assuming that parameters appearing in the cell drag and pressure terms, namely $\psi_{\mathrm{ns}}, \nu, \delta_{\mathrm{a}}$, and $\gamma_{\mathrm{ns}}$, are the same for each cell population. We note that it would be straightforward to repeat the analysis of this paper with parameters which differ between cell populations.

Results presented here are qualitative in nature, but the model derivation is general and could easily be applied to a specific experimental setup given appropriate parameter values. For a target differentiated cell percentage, this model could be used to optimise operating conditions (specifically, end time, inlet fluid fluxes, and inlet growth factor concentration) to achieve the required quantity of differentiated cells in an efficient manner, e.g. by minimising the amount of growth factor needed to reduce costs.

\section{Notes}

\footnotetext{
${ }^{1}$ http://www.lifetechnologies.com/order/catalog/product/ PHC7141?ICID = searchproduct.

${ }^{2}$ Personal communication with Dr Marianne Ellis, Centre for Regenerative Medicine, University of Bath.

${ }^{3}$ We note that the values for $D$ and $C^{*}$ are for different growth factors; this is due to a scarcity of data as these parameters are rarely quantified experimentally.
}

Acknowledgements N.C.P. is funded by an Engineering and Physical Sciences Research Council (EPSRC) studentship through the Systems Biology Doctoral Training Centre at the University of Oxford.

\section{A Dimensionless equations}

Following the non-dimensionalisation in $\S 3.2$, we present the dimensionless equations in the remaining layers of the modelling domain. Beginning in the lumen, we have

$$
\begin{gathered}
\frac{\partial u_{1}}{\partial x}+\frac{\partial v_{1}}{\partial y}=0, \quad-\frac{\partial p_{1}}{\partial x}+\varepsilon^{2} \frac{\partial^{2} u_{1}}{\partial x^{2}}+\frac{\partial^{2} u_{1}}{\partial y^{2}}=0 \\
-\frac{\partial p_{1}}{\partial y}+\varepsilon^{4} \frac{\partial^{2} v_{1}}{\partial x^{2}}+\varepsilon^{2} \frac{\partial^{2} v_{1}}{\partial y^{2}}=0 \\
\varepsilon^{2} \operatorname{Pe}\left(\lambda \varepsilon \frac{\partial c_{1}}{\partial t}+\nabla \cdot\left(c_{1} \mathbf{u}_{1}\right)\right)=\varepsilon^{2} \frac{\partial^{2} c_{1}}{\partial x^{2}}+\frac{\partial^{2} c_{1}}{\partial y^{2}}
\end{gathered}
$$


In the porous membrane, the dimensionless equations are

$$
\begin{gathered}
u_{\mathrm{m}}=-\varepsilon^{2} \kappa_{\mathrm{m}} \frac{\partial p_{\mathrm{m}}}{\partial x}, \quad v_{\mathrm{m}}=-\kappa_{\mathrm{m}} \frac{\partial p_{\mathrm{m}}}{\partial y} \\
\varepsilon^{2} \frac{\partial^{2} p_{\mathrm{m}}}{\partial x^{2}}+\frac{\partial^{2} p_{\mathrm{m}}}{\partial y^{2}}=0 \\
\lambda \varepsilon^{3} \operatorname{Pe}\left(\frac{\partial c_{\mathrm{m}}}{\partial t}+\nabla \cdot\left(c_{\mathrm{m}} \mathbf{u}_{\mathrm{m}}\right)\right)=\varepsilon^{2} \frac{\partial^{2} c_{\mathrm{m}}}{\partial x^{2}}+\frac{\partial^{2} c_{\mathrm{m}}}{\partial y^{2}},
\end{gathered}
$$

where $\kappa=k /\left(\lambda \varepsilon^{5} L^{2}\right)$ is the $\mathrm{O}(1)$ dimensionless permeability (see Table 3). Finally, in the 4-layer model the upper fluid layer equations are

$$
\begin{gathered}
\frac{\partial u_{\mathrm{f}}}{\partial x}+\frac{\partial v_{\mathrm{f}}}{\partial y}=0, \quad-\frac{\partial p_{\mathrm{f}}}{\partial x}+\varepsilon^{2} \frac{\partial^{2} u_{\mathrm{f}}}{\partial x^{2}}+\frac{\partial^{2} u_{\mathrm{f}}}{\partial y^{2}}=0 \\
-\frac{\partial p_{\mathrm{f}}}{\partial y}+\varepsilon^{4} \frac{\partial^{2} v_{\mathrm{f}}}{\partial x^{2}}+\varepsilon^{2} \frac{\partial^{2} v_{\mathrm{f}}}{\partial y^{2}}=0 \\
\varepsilon^{2} \operatorname{Pe}\left(\lambda \varepsilon \frac{\partial c_{\mathrm{f}}}{\partial t}+\nabla \cdot\left(c_{\mathrm{f}} \mathbf{u}_{\mathrm{f}}\right)\right)=\varepsilon^{2} \frac{\partial^{2} c_{\mathrm{f}}}{\partial x^{2}}+\frac{\partial^{2} c_{\mathrm{f}}}{\partial y^{2}}
\end{gathered}
$$

\section{References}

Abdullah N S, Das D B (2007) Modelling nutrient transport in hollow fibre membrane bioreactor for growing bone tissue with consideration of multi-component interactions. Chem Eng Sci 62(21):5821-5839. doi:10.1016/j.ces.2007.06.024

Arnsdorf E J, Tummala P, Kwon R Y, Jacobs C R (2009) Mechanically induced osteogenic differentiation - the role of RhoA, ROCKII and cytoskeletal dynamics. J Cell Sci 122(4):546553. doi: $10.1242 /$ jcs.036293

Breier G, Albrecht U, Sterrer S, Risau W (1992) Expression of vascular endothelial growth factor during embryonic angiogenesis and endothelial cell differentiation. Development 114(2):521-532.

Brouwers J E M, van Donkelaar C C, Sengers B G, Huiskes R (2006) Can the growth factors PTHrP, Ihh and VEGF, together regulate the development of a long bone? J Biomech 39(15):2774-2782. doi:10.1016/j.jbiomech.2005.10.004

Byrne H M, Owen M R, Alarcon T, Murphy J, Maini P K (2006) Modelling the response of vascular tumours to chemotherapy: A multiscale approach. Math Models Methods Appl Sci 16(7S):1219-1241. doi:10.1142/S0218202506001522

Gramer M J, Poeschl D M (2000) Comparison of cell growth in Tflasks, in micro hollow fiber bioreactors, and in an industrial scale hollow fiber bioreactor system. Cytotechnology 34(12):111-119. doi:10.1023/A:1008167713696

Kapur S, Baylink D J, Lau K-H W (2003) Fluid flow shear stress stimulates human osteoblast proliferation and differentiation through multiple interacting and competing signal transduction pathways. Bone 32(3):241-251. doi:10.1016/S87563282(02)00979-1

Keane J T, Ryan D, Gray P P (2003) Effect of shear stress on expression of a recombinant protein by chinese hamster ovary cells. Biotech. Boiling. 81:211-220.

Knazek R A, Gullino $\mathrm{P}$ M, Kohler $\mathrm{P}$ O, Dedrick $\mathrm{R}$ L (1972) Cell Culture on Artificial Capillaries: An Approach to Tissue Growth in vitro. Science 178(4056):65-67. doi:10.1126/science.178.4056.65

Knazek R A, Kohler P O, Gullino P M (1974) Hormone production by cells grown in vitro on artificial capillaries. Exp Cell Res 84(1-2):251-254. doi:10.1016/0014-4827(74)90403-0

Korin N, Bransky A, Dinnar U, Levenberg S (2009) Periodic "flow-stop" perfusion micro channel bioreactors for mammalian and human embryonic stem cell long-term culture. Boomed. Microwave. 11: 87-94.]
Lemon G, King J R, Byrne H M, Jensen O E, Shakesheff K M (2006) Mathematical modelling of engineered tissue growth using a multiphase porous flow mixture theory. J Math Biol 52:571-594. doi:10.1007/s00285-005-0363-1

Li W-J, Tuli R, Okafor C, Derfoul A, Danielson K G, Hall D J, Tuan R S (2005) A three-dimensional nanofibrous scaffold for cartilage tissue engineering using human mesenchymal stem cells. Biomaterials 26(6):599-609. doi:10.1016/j.biomaterials.2004.03.005

Mac Gabhann F, Yang M T, Popel A S (2005) Monte Carlo simulations of VEGF binding to cell surface receptors in vitro. Biochim Biophys Acta Mol Cell Res 1746(2):95-107. doi:10.1016/j.bbamcr.2005.09.004

Martin I, Wendt D, Heberer M (2004) The role of bioreactors in tissue engineering. Trends Biotechnol 22(2):80-86. doi:10.1016/j.tibtech.2003.12.001

Martin Y, Vermette P (2005) Bioreactors for tissue mass culture: Design, characterization, and recent advances. Biomaterials 26(35):7481-7503. doi:10.1016/j.biomaterials.2005.05.057

Mayer H, Bertram H, Lindenmaier W, Korff T, Weber H, Weich H (2005) Vascular endothelial growth factor (VEGF-A) expression in human mesenchymal stem cells: Autocrine and paracrine role on osteoblastic and endothelial differentiation. J Cell Biochem 95(4):827-839. doi:10.1002/jcb.20462

Meneghello G, Parker D J, Ainsworth B J, Perera S P, Chaudhuri J B, Ellis M J, De Bank P A (2009) Fabrication and characterization of poly(lactic-co-glycolic acid)/polyvinyl alcohol blended hollow fibre membranes for tissue engineering applications. J Membr Sci 344(1-2):55-61. doi:10.1016/j.memsci.2009.07.034

Midy V, Plouet J (1994) Vasculotropin/vascular endothelial growth factor induces differentiation in cultured osteoblasts. Biochem Biophys Res Commun 199(1):380-386. doi:10.1006/bbrc.1994.1240

Mohebbi-Kalhori D, Behzadmehr A, Doillon C J, Hadjizadeh A (2012) Computational modeling of adherent cell growth in a hollow-fiber membrane bioreactor for large-scale 3D bone tissue engineering. J Artif Organs 15:250-265. doi:10.1007/s10047-012-0649-1

Obi S, Yamamoto K, Shimizu N, Kumagaya S, Masumura T, Sokabe T, Asahara T, Ando J (2009) Fluid shear stress induces arterial differentiation of endothelial progenitor cells. J Appl Physiol 106(1):203-211. doi:10.1152/japplphysiol.00197.2008

O'Dea R D, Waters S L, Byrne H M (2010) A multiphase model for tissue construct growth in a perfusion bioreactor. Math Med Biol 27(2):95-127. doi:10.1093/imammb/dqp003

Park J, Li Y, Berthiaume F, Toner M, Yarmouth M L, Tilles A W (2008) Radial flow hepatocyte bioreactor using stacked microfabricated grooved substrates. Biotech. Bioeng. 99: 455467.

Pearson N C, Shipley R J, Waters S L, Oliver J M (2013) Multiphase modelling of the influence of fluid flow and chemical concentration on tissue growth in a hollow fibre membrane bioreactor. Math Med Biol. doi:10.1093/imammb/dqt015

Pearson N C, Waters S L, Oliver J M, Shipley R J (2014) Multiphase modelling of the effect of fluid shear stress on cell yield and distribution in a hollow fibre membrane bioreactor. Biomech Model Mechan pages 1-16. doi:10.1007/s10237-0140611-7

Pearson N C, Shipley R J, Waters S L, Oliver J M (2014a) Dispersion-enhanced solute transport in a cell-seeded hollow fibre membrane bioreactor. Submitted.

Peters K G, De Vries C, Williams L T (1993) Vascular endothelial growth factor receptor expression during embryogenesis and tissue repair suggests a role in endothelial differentiation and blood vessel growth. Proc Natl Acad Sci USA 90(19):8915- 
8919.

Planchamp C, Vu T L, Mayer J M, Reist M, Testa B (2003) Hepatocyte hollow-fibre bioreactors: Design, set-up, validation and applications. J Pharm Pharmacol 55(9):1181-1198. doi:10.1211/0022357021963

Plank M J, Sleeman B D, Jones P F (2004) A mathematical model of tumour angiogenesis, regulated by vascular endothelial growth factor and the angiopoietins. J Theor Biol 229(4):435-454. doi:10.1016/j.jtbi.2004.04.012

Prabhakaran M P, Venugopal J R, Ramakrishna S (2009) Mesenchymal stem cell differentiation to neuronal cells on electrospun nanofibrous substrates for nerve tissue engineering. Biomaterials 30(28):4996-5003. doi:10.1016/j.biomaterials.2009.05.057

Shipley R J, Waters S L, Ellis M J (2010) Definition and validation of operating equations for poly(vinyl alcohol)-poly(lactide-co-glycolide) microfiltration membranescaffold bioreactors. Biotechnol Bioeng 107(2):382-392. doi:10.1002/bit.22815

Shipley R J, Davidson A J, Chan K, Chaudhuri J B, Waters S L, Ellis M J (2011) A strategy to determine operating parameters in tissue engineering hollow fiber bioreactors. Biotechnol Bioeng 108(6):1450-1461. doi:10.1002/bit.23062

Shipley R J, Waters S L (2012) Fluid and mass transport modelling to drive the design of cell-packed hollow fibre bioreactors for tissue engineering applications. Math Med Biol 29:329-359. doi:10.1093/imammb/dqr025

Tharakan J P, Chau P C (1986) A radial flow hollow fiber bioreactor for the large-scale culture of mammalian cells. Biotechnol Bioeng 28(3):329-342. doi:10.1002/bit.260280305

Tourovskaia A, Figueroa-Masot X, Folch A (2005) Differentiation-on-a-chip: A microfluidic platform for long-term cell culture studies. Lab Chip. 5:14-19.

Wu C-C, Chao Y-C, Chen C-N, Chien S, Chen Y-C, Chien CC, Chiu J-J, Yen B L (2008) Synergism of biochemical and mechanical stimuli in the differentiation of human placentaderived multipotent cells into endothelial cells. J Biomech 41(4):813-821. doi:10.1016/j.jbiomech.2007.11.008

Yamamoto K, Sokabe T, Watabe T, Miyazono K, Yamashita J K, Obi S, Ohura N, Matsushita A, Kamiya A, Ando J (2005) Fluid shear stress induces differentiation of Flk-1positive embryonic stem cells into vascular endothelial cells in vitro. Am J Physiol Heart Circ Physiol 288(4):H1915-H1924. doi:10.1152/ajpheart.00956.2004

Ye H, Das D B, Triffitt J T, Cui Z (2006) Modelling nutrient transport in hollow fibre membrane bioreactors for growing three-dimensional bone tissue. J Membr Sci 272(1-2):169-178. doi:10.1016/j.memsci.2005.07.040

Ye H, Xia Z, Ferguson D J P, Triffitt J T, Cui Z (2007) Studies on the use of hollow fibre membrane bioreactors for tissue generation by using rat bone marrow fibroblastic cells and a composite scaffold. J Mater Sci Mater Med 18(4):641-648. doi:10.1007/s10856-007-2314-4 